\title{
Characteristic Components and Authenticity Evaluation of Rape, Acacia, and Linden Honey
}

Jiangtao Qiao ${ }^{a}$, Lihong Chen, ${ }^{a, c}$, Lingjie Konga, Jie Dong ${ }^{a}$, Zhuoqiang Zhou $^{\text {b* }}$, Hongcheng Zhang ${ }^{\text {a* }}$

${ }^{a}$ Institute of Apicultural Research, Chinese Academy of Agricultural Sciences, Beijing, 100093, China

${ }^{b}$ College of Materials and Energy, South China Agricultural University, Guangzhou, 510640, China

${ }^{c}$ Apicultural Science Association of China, Beijing, 100081, China

*Corresponding author: Tel: +86 1062590442 ;

Fax: +86 10 62590442;

Email: 460414874@qq.com (H. Zhang) zqzhou@scau.edu.cn (Z. Zhou)

Address: Institute of Apicultural Research, Chinese Academy of Agricultural Sciences, Xiangshan, Beijing, 100093, China 
Supplementary Tab 1. Characterization of the analyzed honey samples

\begin{tabular}{|c|c|c|c|c|c|}
\hline Brands & Type of honey & Botanical origin & Predominant pollen $(\%)$ & Geographical origin & Production year \\
\hline A 1 & Rape honey & Brassica napus L. & 82 & Yunnan & 2018 \\
\hline A 2 & Rape honey & Brassica napus L. & 78 & Yunnan & 2018 \\
\hline A 3 & Rape honey & Brassica napus L. & 79 & Yunnan & 2018 \\
\hline A 4 & Rape honey & Brassica napus L. & 88 & Yunnan & 2018 \\
\hline A 5 & Rape honey & Brassica napus L. & 81 & Qinghai & 2018 \\
\hline A 6 & Rape honey & Brassica napus L. & 84 & Qinghai & 2018 \\
\hline A 7 & Rape honey & Brassica napus L. & 77 & Hubei & 2018 \\
\hline A 8 & Rape honey & Brassica napus L. & 89 & Hubei & 2018 \\
\hline A 9 & Rape honey & Brassica napus L. & 72 & Hebei & 2018 \\
\hline A 10 & Rape honey & Brassica napus L. & 88 & Hebei & 2018 \\
\hline B 1 & Acacia honey & Robinia pseudacacia L. & 79 & Gansu & 2017 \\
\hline B 2 & Acacia honey & Robinia pseudacacia L. & 82 & Gansu & 2017 \\
\hline B 3 & Acacia honey & Robinia pseudacacia L. & 76 & Gansu & 2017 \\
\hline B 4 & Acacia honey & Robinia pseudacacia L. & 75 & Gansu & 2018 \\
\hline B 5 & Acacia honey & Robinia pseudacacia L. & 84 & Gansu & 2018 \\
\hline B 6 & Acacia honey & Robinia pseudacacia L. & 81 & Gansu & 2018 \\
\hline B 7 & Acacia honey & Robinia pseudacacia L. & 73 & Shanxi & 2017 \\
\hline B 8 & Acacia honey & Robinia pseudacacia L. & 87 & Shanxi & 2017 \\
\hline B 9 & Acacia honey & Robinia pseudacacia L. & 73 & Shanxi & 2018 \\
\hline
\end{tabular}




\begin{tabular}{|c|c|c|c|c|c|}
\hline Brands & Type of honey & Botanical origin & Predominant pollen $(\%)$ & Geographical origin & Production year \\
\hline B 10 & Acacia honey & Robinia pseudacacia L. & 82 & Shanxi & 2018 \\
\hline B 11 & Acacia honey & Robinia pseudacacia L. & 88 & Shaanxi & 2017 \\
\hline B 12 & Acacia honey & Robinia pseudacacia L. & 77 & Shaanxi & 2017 \\
\hline В 13 & Acacia honey & Robinia pseudacacia L. & 75 & Shaanxi & 2017 \\
\hline B 14 & Acacia honey & Robinia pseudacacia L. & 82 & Shaanxi & 2018 \\
\hline B 15 & Acacia honey & Robinia pseudacacia L. & 87 & Shaanxi & 2018 \\
\hline B 16 & Acacia honey & Robinia pseudacacia L. & 79 & Shaanxi & 2018 \\
\hline B 17 & Acacia honey & Robinia pseudacacia L. & 79 & Henan & 2017 \\
\hline B 18 & Acacia honey & Robinia pseudacacia L. & 82 & Henan & 2017 \\
\hline B 19 & Acacia honey & Robinia pseudacacia L. & 88 & Henan & 2018 \\
\hline B 20 & Acacia honey & Robinia pseudacacia L. & 74 & Henan & 2018 \\
\hline В 21 & Acacia honey & Robinia pseudacacia L. & 73 & Jiangsu & 2017 \\
\hline B 22 & Acacia honey & Robinia pseudacacia L. & 89 & Jiangsu & 2017 \\
\hline В 23 & Acacia honey & Robinia pseudacacia L. & 81 & Hebei & 2017 \\
\hline B 24 & Acacia honey & Robinia pseudacacia L. & 85 & Hebei & 2017 \\
\hline B 25 & Acacia honey & Robinia pseudacacia L. & 77 & Hebei & 2018 \\
\hline B 26 & Acacia honey & Robinia pseudacacia L. & 80 & Hebei & 2018 \\
\hline B 27 & Acacia honey & Robinia pseudacacia L. & 79 & Shandong & 2017 \\
\hline B 28 & Acacia honey & Robinia pseudacacia L. & 82 & Shandong & 2017 \\
\hline B 29 & Acacia honey & Robinia pseudacacia L. & 88 & Beijing & 2017 \\
\hline
\end{tabular}




\begin{tabular}{|c|c|c|c|c|c|}
\hline Brands & Type of honey & Botanical origin & Predominant pollen (\%) & Geographical origin & Production year \\
\hline B 30 & Acacia honey & Robinia pseudacacia $\mathrm{L}$. & 82 & Beijing & 2017 \\
\hline B 31 & Acacia honey & Robinia pseudacacia $\mathrm{L}$. & 82 & Beijing & 2018 \\
\hline B 32 & Acacia honey & Robinia pseudacacia $\mathrm{L}$. & 74 & Beijing & 2018 \\
\hline B 33 & Acacia honey & Robinia pseudacacia $\mathrm{L}$. & 72 & Rigao Co., Ltd & 2017 \\
\hline B 34 & Acacia honey & Robinia pseudacacia $\mathrm{L}$. & 71 & Rigao Co., Ltd & 2017 \\
\hline B 35 & Acacia honey & Robinia pseudacacia $\mathrm{L}$. & 67 & Rigao Co., Ltd & 2017 \\
\hline B 36 & Acacia honey & Robinia pseudacacia $\mathrm{L}$. & 65 & Rigao Co., Ltd & 2017 \\
\hline B 37 & Acacia honey & Robinia pseudacacia $\mathrm{L}$. & 74 & Mingyuan Co., Ltd & 2017 \\
\hline B 38 & Acacia honey & Robinia pseudacacia $\mathrm{L}$. & 68 & Mingyuan Co., Ltd & 2017 \\
\hline B 39 & Acacia honey & Robinia pseudacacia $\mathrm{L}$. & 63 & Mingyuan Co., Ltd & 2017 \\
\hline B 40 & Acacia honey & Robinia pseudacacia $\mathrm{L}$. & 69 & Mingyuan Co., Ltd & 2017 \\
\hline $\mathrm{C} 1$ & Linden honey & Tilia amurensis Rupr. & 73 & Heilongjiang & 2018 \\
\hline $\mathrm{C} 2$ & Linden honey & Tilia amurensis Rupr. & 71 & Heilongjiang & 2018 \\
\hline $\mathrm{C} 3$ & Linden honey & Tilia amurensis Rupr. & 79 & Heilongjiang & 2018 \\
\hline $\mathrm{C} 4$ & Linden honey & Tilia amurensis Rupr. & 82 & Heilongjiang & 2018 \\
\hline C 5 & Linden honey & Tilia amurensis Rupr. & 88 & Heilongiiang & 2018 \\
\hline $\mathrm{C} 6$ & Linden honey & Tilia amurensis Rupr. & 79 & Heilongiiang & 2018 \\
\hline C 7 & Linden honey & Tilia amurensis Rupr. & 89 & Heilongjiang & 2018 \\
\hline $\mathrm{C} 8$ & Linden honey & Tilia amurensis Rupr. & 73 & Heilongjiang & 2018 \\
\hline C 9 & Linden honey & Tilia amurensis Rupr. & 75 & Heilongjiang & 2019 \\
\hline
\end{tabular}




\begin{tabular}{|c|c|c|c|c|c|}
\hline Brands & Type of honey & Botanical origin & Predominant pollen (\%) & Geographical origin & Production year \\
\hline C 10 & Linden honey & Tilia amurensis Rupr. & 78 & Heilongjiang & 2019 \\
\hline C 11 & Linden honey & Tilia amurensis Rupr. & 80 & Heilongjiang & 2019 \\
\hline C 12 & Linden honey & Tilia amurensis Rupr. & 89 & Heilongjiang & 2019 \\
\hline C 13 & Linden honey & Tilia amurensis Rupr. & 72 & Jilin & 2018 \\
\hline C 14 & Linden honey & Tilia amurensis Rupr. & 79 & Jilin & 2018 \\
\hline C 15 & Linden honey & Tilia amurensis Rupr. & 88 & Jilin & 2018 \\
\hline C 16 & Linden honey & Tilia amurensis Rupr. & 78 & Jilin & 2018 \\
\hline C 17 & Linden honey & Tilia amurensis Rupr. & 79 & Jilin & 2018 \\
\hline C 18 & Linden honey & Tilia amurensis Rupr. & 80 & Jilin & 2018 \\
\hline C 19 & Linden honey & Tilia amurensis Rupr. & 81 & Jilin & 2019 \\
\hline C 20 & Linden honey & Tilia amurensis Rupr. & 82 & Jilin & 2019 \\
\hline C 21 & Linden honey & Tilia amurensis Rupr. & 89 & Jilin & 2019 \\
\hline C 22 & Linden honey & Tilia amurensis Rupr. & 82 & Jilin & 2019 \\
\hline C 23 & Linden honey & Tilia amurensis Rupr. & 71 & Jilin & 2019 \\
\hline C 24 & Linden honey & Tilia amurensis Rupr. & 74 & Jilin & 2019 \\
\hline C 25 & Linden honey & Tilia amurensis Rupr. & 70 & Liaoning & 2018 \\
\hline C 26 & Linden honey & Tilia amurensis Rupr. & 88 & Liaoning & 2018 \\
\hline C 27 & Linden honey & Tilia amurensis Rupr. & 74 & Liaoning & 2018 \\
\hline C 28 & Linden honey & Tilia amurensis Rupr. & 89 & Liaoning & 2018 \\
\hline C 29 & Linden honey & Tilia amurensis Rupr. & 80 & Liaoning & 2019 \\
\hline
\end{tabular}




\begin{tabular}{|c|c|c|c|c|c|}
\hline Brands & Type of honey & Botanical origin & Predominant pollen $(\%)$ & Geographical origin & Production year \\
\hline B 30 & Linden honey & Tilia amurensis Rupr. & 87 & Liaoning & 2019 \\
\hline B 31 & Linden honey & Tilia amurensis Rupr. & 65 & Senfengyuan Co., Ltd & 2018 \\
\hline B 32 & Linden honey & Tilia amurensis Rupr. & 65 & Senfengyuan Co., Ltd & 2018 \\
\hline В 33 & Linden honey & Tilia amurensis Rupr. & 68 & Senfengyuan Co., Ltd & 2018 \\
\hline B 34 & Linden honey & Tilia amurensis Rupr. & 73 & Senfengyuan Co., Ltd & 2018 \\
\hline В 35 & Linden honey & Tilia amurensis Rupr. & 78 & Senfengyuan Co., Ltd & 2018 \\
\hline В 36 & Linden honey & Tilia amurensis Rupr. & 70 & Senfengyuan Co., Ltd & 2018 \\
\hline В 37 & Linden honey & Tilia amurensis Rupr. & 71 & Mingyuan Co., Ltd & 2018 \\
\hline B 38 & Linden honey & Tilia amurensis Rupr. & 75 & Mingyuan Co., Ltd & 2018 \\
\hline В 39 & Linden honey & Tilia amurensis Rupr. & 70 & Mingyuan Co., Ltd & 2018 \\
\hline B 40 & Linden honey & Tilia amurensis Rupr. & 72 & Mingyuan Co., Ltd & 2018 \\
\hline
\end{tabular}


Supplementary Tab 2. Manufacturing date and address of rape, acacia and linden honeys

\begin{tabular}{ccc}
\hline Brands & Date & Address \\
\hline D 1 & $06 / 03 / 2019$ & Shanghai \\
D 2 & $16 / 04 / 2019$ & Jiangsu \\
D 3 & $09 / 05 / 2019$ & Hunan \\
D 4 & $14 / 03 / 2019$ & Qinghai \\
D 5 & $22 / 03 / 2019$ & Beijing \\
D 6 & $21 / 04 / 2019$ & Hubei \\
E 1 & $17 / 10 / 2018$ & Jiangsu \\
E 2 & $13 / 11 / 2018$ & Shandong \\
E 3 & $22 / 01 / 2019$ & Beijing \\
E 4 & $06 / 04 / 2019$ & Shandong \\
E 5 & $19 / 03 / 2019$ & Shanghai \\
E 6 & $07 / 05 / 2019$ & Hunan \\
E 7 & $11 / 10 / 2018$ & Heilongjiang \\
E 8 & $07 / 01 / 2019$ & Zhejiang \\
E 9 & $08 / 06 / 2018$ & Beijing \\
E 10 & $12 / 07 / 2018$ & Nanjing \\
F 1 & $17 / 09 / 2018$ & Beijing \\
F 2 & $08 / 01 / 2018$ & Shanghai \\
F 3 & $24 / 08 / 2018$ & Jiangsu \\
F 4 & $21 / 11 / 2018$ & Heilongjiang \\
F 5 & $13 / 07 / 2018$ & Guangdong \\
F 6 & $23 / 10 / 2018$ & Jilin \\
F 7 & $27 / 09 / 2018$ & Hunan \\
F 8 & $18 / 03 / 2018$ & Zhejiang \\
F 9 & $04 / 08 / 2018$ & Henan \\
F 10 & $17 / 08 / 2018$ & Shanghai \\
\hline & &
\end{tabular}


Supplementary Tab. 3. Phytochemical contents in raw rape honey samples $(\mathrm{mg} / \mathrm{kg})$

\begin{tabular}{|c|c|c|c|c|c|c|c|c|c|c|}
\hline Compounds & A 01 & A 02 & A 03 & A 04 & A 05 & A 06 & A 07 & A 08 & A 09 & A 10 \\
\hline Vanilllic acid & 1.29 & 1.36 & 1.29 & 1.02 & 1.07 & 1.21 & 0.74 & 0.83 & 1.86 & 2.38 \\
\hline p-Coumaric acid & 0.58 & 0.59 & 0.54 & 1.13 & 0.61 & 0.84 & 1.16 & 1.11 & 0.52 & 0.64 \\
\hline trans, trans -abscisic acid & 0.34 & 0.36 & 0.33 & 1.03 & 1.19 & 0.96 & 0.77 & 0.78 & 0.46 & 0.46 \\
\hline Pinobanksin & 0.56 & 0.56 & 0.67 & 0.70 & 0.55 & 0.65 & 0.63 & 0.64 & 0.61 & 0.63 \\
\hline Kaempferol & 0.53 & 0.59 & 0.59 & 1.01 & 0.66 & 0.82 & 0.77 & 0.81 & 0.65 & 0.68 \\
\hline
\end{tabular}

Note. Average calculated from two independent analyses. (一): not detected. 
Supplementary Tab. 4. Phytochemical contents in raw acacia honey samples $(\mathrm{mg} / \mathrm{kg})$

\begin{tabular}{|c|c|c|c|c|c|c|c|c|c|c|}
\hline Compounds & B 01 & В 02 & В 03 & В 04 & В 05 & В 06 & В 07 & В 08 & В 09 & B 10 \\
\hline 4-Hydroxy benzoic acid & 0.64 & - & 0.60 & - & - & - & 0.66 & - & - & 0.75 \\
\hline Vanilllic acid & 0.25 & 0.30 & - & - & - & - & - & - & - & - \\
\hline p-Coumaric acid & 0.10 & 0.21 & - & 0.06 & - & 0.13 & - & - & - & 0.16 \\
\hline Benzoic acid & - & - & 0.89 & - & - & - & - & - & - & - \\
\hline Ferulic acid & 0.25 & 0.24 & - & - & - & 0.19 & - & - & - & 0.22 \\
\hline Phaseic acid & 0.59 & 1.41 & 0.87 & 0.59 & 0.37 & 0.68 & 0.44 & 0.59 & 0.60 & 0.79 \\
\hline Isoferulic acid & 0.15 & 0.13 & - & - & - & 0.05 & - & - & - & - \\
\hline Methyl syringate & 0.24 & - & 0.01 & 0.07 & - & - & 0.04 & - & - & - \\
\hline trans, trans -abscisic acid & 0.10 & 0.59 & 0.21 & 0.26 & 0.11 & 0.16 & 0.11 & 0.10 & 0.10 & 0.77 \\
\hline K-3-O-Rob-7-O-Rh & 0.28 & 0.33 & 0.37 & - & 0.40 & 0.38 & 0.36 & 0.21 & 0.26 & 0.73 \\
\hline cis, trans-abscisic acid & 3.28 & 3.21 & 2.89 & 1.95 & 1.99 & 2.09 & 2.05 & 1.24 & 2.18 & 2.73 \\
\hline Cinnamic acid & 0.01 & 0.01 & 0.01 & 0.01 & - & 0.02 & 0.01 & 0.01 & - & - \\
\hline Pinobanksin & 0.77 & 0.41 & 0.16 & - & 0.44 & 0.33 & 0.56 & - & 0.10 & - \\
\hline Alpinetin & 0.07 & - & 0.02 & - & - & - & 0.09 & 0.05 & - & - \\
\hline Kaempferol & 0.15 & 0.05 & 0.03 & 0.07 & 0.04 & - & - & - & 0.11 & - \\
\hline Pinocembrin & 0.50 & 0.21 & 0.12 & 0.11 & 0.24 & 0.23 & 0.25 & 0.11 & 0.09 & - \\
\hline Benzyl caffeate & 0.33 & 0.95 & - & - & - & - & - & - & - & - \\
\hline Pinobanksin-3-O-acetate & 0.40 & - & - & - & - & 0.32 & 0.27 & - & - & - \\
\hline Chrysin & 0.86 & 0.46 & 0.36 & 0.37 & 0.59 & - & 0.70 & - & 0.34 & - \\
\hline Phenethyl caffeate & 0.28 & 0.08 & - & - & - & 一 & 一 & - & 一 & - \\
\hline Galangin & 0.42 & 0.28 & 0.23 & - & - & 0.50 & 0.33 & - & - & - \\
\hline
\end{tabular}




\begin{tabular}{|c|c|c|c|c|c|c|c|c|c|c|}
\hline Compounds & B 11 & B 12 & B 13 & B 14 & B 15 & B 16 & B 17 & B 18 & B 19 & B 20 \\
\hline 4-hydroxy benzoic acid & 0.63 & - & - & - & - & 0.69 & - & 0.80 & 0.71 & 0.73 \\
\hline Vanilllic acid & 0.24 & - & - & - & - & 0.27 & - & - & - & - \\
\hline Caffeic acid & 0.04 & - & - & - & - & - & - & 0.35 & 0.08 & - \\
\hline p-Coumaric acid & 0.09 & - & - & 0.09 & - & 0.04 & - & 0.21 & 0.08 & 0.10 \\
\hline Benzoic acid & - & - & - & - & - & - & - & - & - & - \\
\hline Ferulic acid & 0.17 & - & - & - & 0.09 & 0.05 & 0.10 & - & - & 0.23 \\
\hline Phaseic acid & 0.67 & 0.33 & 0.90 & 1.14 & 0.61 & 0.33 & 0.38 & 0.62 & 0.33 & 0.30 \\
\hline Isoferulic acid & 0.06 & - & - & - & - & 0.11 & - & - & - & - \\
\hline Methyl syringate & - & - & - & - & - & 0.01 & 0.00 & - & - & - \\
\hline trans, trans -abscisic acid & 0.54 & 0.21 & 0.03 & 0.18 & 0.11 & 0.05 & 0.12 & 0.12 & 0.06 & 0.34 \\
\hline K-3-O-Rob-7-O-Rh & 0.46 & 0.34 & - & 0.51 & 0.22 & 0.20 & 0.30 & - & 0.61 & 0.81 \\
\hline cis, trans-abscisic acid & 1.80 & 1.82 & 1.98 & 3.53 & 1.25 & 1.05 & 1.60 & 3.17 & 1.61 & 1.98 \\
\hline Cinnamic acid & 0.01 & 0.01 & - & - & - & 0.01 & - & - & - & 0.01 \\
\hline 5-methoxy pinobanksin & 0.33 & 0.40 & - & - & 0.36 & 0.39 & - & 一 & 0.36 & - \\
\hline Pinobanksin & 0.21 & - & 0.36 & 0.34 & - & 0.32 & 0.10 & 0.29 & - & 0.13 \\
\hline Alpinetin & - & - & - & - & 0.10 & 0.10 & - & - & - & 0.18 \\
\hline Kaempferol & 0.05 & 0.05 & 一 & 一 & 0.05 & 0.05 & 一 & 0.25 & 0.41 & 0.06 \\
\hline Pinocembrin & 0.15 & - & 0.34 & 0.22 & 0.10 & 0.18 & - & 0.16 & 0.11 & 0.11 \\
\hline Benzyl caffeate & - & - & 一 & 一 & 一 & 0.12 & 一 & - & 一 & - \\
\hline Pinobanksin-3-O-acetate & - & - & 0.37 & - & - & 0.18 & - & - & - & - \\
\hline Chrysin & 0.43 & - & 0.87 & 0.44 & 0.47 & 0.50 & - & 0.42 & 0.50 & 0.34 \\
\hline Phenethyl caffeate & - & 0.45 & - & - & - & 0.16 & - & - & - & - \\
\hline Galangin & 0.26 & - & 0.62 & - & - & 0.24 & - & 0.26 & - & - \\
\hline
\end{tabular}




\begin{tabular}{ccccccccccc}
\hline Compounds & B 21 & B 22 & B 23 & B 24 & B 25 & B 26 & B 27 & B 28 & B 29 & B 30 \\
\hline 4-hydroxy benzoic acid & - & - & 0.77 & 0.68 & 0.59 & - & - & 0.73 & - & 0.63 \\
Vanillic acid & - & - & - & - & - & - & - & - & - & - \\
Caffeic acid & - & - & - & - & 0.04 & - & 0.06 & 0.07 & - & - \\
p-Coumaric acid & 0.11 & - & - & - & 0.11 & - & - & 0.10 & - & - \\
Benzoic acid & - & - & - & - & - & - & - & - & - & - \\
Ferulic acid & - & 0.10 & 0.10 & 0.12 & 0.38 & - & - & 0.06 & - & 0.09 \\
Phaseic acid & 0.83 & 0.32 & 0.31 & 0.42 & 0.33 & 1.93 & 0.33 & 0.42 & 0.36 & 0.37 \\
Isoferulic acid & - & 0.04 & - & - & - & - & 0.08 & - & - & 0.04 \\
Methyl syringate & 0.18 & 0.01 & - & - & - & 0.54 & 0.20 & - & - & 0.01 \\
trans, trans -abscisic acid & 0.47 & 0.09 & 0.23 & 0.16 & 0.39 & 1.11 & 0.15 & 0.23 & 0.01 & 0.11 \\
K-3-O-Rob-7-O-Rh & - & 0.24 & 0.29 & 0.31 & 0.78 & 0.74 & 0.23 & 0.29 & 0.32 & 0.32 \\
cis, trans-abscisic acid & 3.02 & 1.28 & 1.30 & 1.63 & 1.57 & 6.15 & 1.62 & 1.83 & 1.14 & 1.61 \\
Cinnamic acid & - & - & - & - & 0.01 & - & 0.01 & 0.01 & 0.01 \\
5-methoxy pinobanksin & - & - & - & - & 0.36 & 0.49 & 0.37 & 0.33 & 0.33 \\
Pinobanksin & 0.16 & 0.09 & 0.10 & 0.08 & 0.20 & - & - & 0.20 & 0.18 & 0.10 \\
Alpinetin & - & - & - & - & 0.34 & - & 0.06 & - & - \\
Kaempferol & 0.07 & 0.08 & 0.10 & 0.09 & - & 0.13 & - & - & 0.10 & 0.11 \\
Pinocembrin & 0.12 & 0.10 & 0.11 & 0.10 & 0.13 & 0.19 & 0.11 & 0.40 & 0.20 & 0.10 \\
Benzyl caffeate & - & 0.16 & 0.09 & 0.05 & 0.92 & - & - & - & - & - \\
Pinobanksin-3-O-acetate & - & - & - & - & - & - & - & 0.59 & 0.19 & - \\
Chrysin & - & - & - & - & 0.38 & 0.45 & 0.49 & 0.97 & 0.43 & - \\
Phenethyl caffeate & - & - & - & - & - & - & - & - & - & - \\
Galangin & 0.28 & 0.29 & 0.29 & 0.27 & - & - & - & 0.78 & 0.31 & 0.26 \\
\hline
\end{tabular}




\begin{tabular}{|c|c|c|c|c|c|c|c|c|c|c|}
\hline Compounds & B 31 & B 32 & B 33 & B 34 & B 35 & B 36 & B 37 & B 38 & B 39 & $\mathrm{~B} 40$ \\
\hline 4-hydroxy benzoic acid & - & 2.00 & - & 0.75 & 0.66 & - & - & 0.97 & 0.70 & 0.68 \\
\hline Vanilllic acid & - & - & - & - & - & - & - & - & - & - \\
\hline Caffeic acid & 0.13 & - & 0.04 & 0.10 & 0.06 & - & - & - & - & - \\
\hline p-Coumaric acid & - & 0.42 & 0.28 & 0.11 & 0.10 & - & - & 0.09 & 0.11 & 0.06 \\
\hline Benzoic acid & - & - & - & 0.99 & 2.22 & - & - & 0.82 & - & - \\
\hline Ferulic acid & - & - & 0.25 & - & 0.22 & - & - & - & - & 0.14 \\
\hline Phaseic acid & 0.88 & 1.10 & 0.91 & 0.58 & 0.40 & 1.88 & 0.34 & 0.39 & 0.30 & 0.44 \\
\hline Isoferulic acid & 0.01 & - & - & - & 0.13 & - & - & - & 0.09 & 0.05 \\
\hline Methyl syringate & 0.70 & - & 0.81 & 0.09 & 0.71 & 0.54 & - & - & 0.00 & 0.00 \\
\hline trans, trans -abscisic acid & 0.31 & 0.27 & 0.31 & 0.29 & 0.31 & 1.10 & 0.10 & 0.00 & 0.23 & 0.09 \\
\hline K-3-O-Rob-7-O-Rh & 0.66 & 0.34 & 0.67 & 0.33 & 0.44 & 0.77 & 0.71 & 0.35 & 0.37 & 0.11 \\
\hline cis, trans-abscisic acid & 3.02 & 1.39 & 2.88 & 1.38 & 1.70 & 6.08 & 1.55 & 1.19 & 1.18 & 1.54 \\
\hline Cinnamic acid & 0.01 & 0.01 & 0.01 & - & - & 0.03 & - & - & - & - \\
\hline 5-methoxy pinobanksin & 0.55 & 0.75 & 0.51 & - & - & 0.45 & 0.43 & 0.43 & - & - \\
\hline Pinobanksin & - & 1.20 & 0.69 & 0.12 & - & - & 0.09 & - & 0.10 & 0.10 \\
\hline Alpinetin & 0.01 & - & - & - & - & - & 0.05 & - & 0.04 & 0.03 \\
\hline Kaempferol & - & - & 0.10 & 0.07 & 0.23 & 0.13 & 0.04 & 0.14 & 0.11 & 0.08 \\
\hline Pinocembrin & 0.30 & - & 0.25 & - & - & 0.19 & - & 0.28 & 0.10 & 0.10 \\
\hline Benzyl caffeate & - & - & - & 0.99 & - & - & - & - & - & - \\
\hline Pinobanksin-3-O-acetate & - & - & - & - & - & - & - & 0.35 & - & - \\
\hline Chrysin & 0.62 & - & 0.55 & - & - & 0.41 & - & 0.66 & - & - \\
\hline Phenethyl caffeate & - & - & - & - & - & - & - & - & - & - \\
\hline Galangin & - & - & 0.29 & - & - & 0.61 & - & 0.48 & 0.28 & 0.26 \\
\hline
\end{tabular}

Note. Average calculated from two independent analyses. (-): not detected. 
Supplementary Tab. 5. Phytochemical contents of in raw linden honey samples $(\mathrm{mg} / \mathrm{kg})$

\begin{tabular}{|c|c|c|c|c|c|c|c|c|c|c|}
\hline Compounds & $\mathrm{C} 01$ & $\mathrm{C} 02$ & $\mathrm{C} 03$ & $\mathrm{C} 04$ & $\mathrm{C} 05$ & $\mathrm{C} 06$ & C 07 & C 08 & C 09 & C 10 \\
\hline p-Coumaric acid & 0.22 & 0.17 & 0.18 & 0.26 & 0.10 & 0.11 & 0.20 & 0.10 & 0.31 & 0.21 \\
\hline Lindenin & 36.63 & 23.73 & 23.76 & 38.05 & 17.27 & 16.26 & 22.67 & 18.33 & 23.86 & 13.30 \\
\hline trans, trans -abscisic acid & 0.49 & 0.43 & 0.61 & 0.26 & 0.36 & 0.49 & 0.48 & 0.49 & 0.47 & 0.43 \\
\hline cis, trans-abscisic acid & 1.97 & 1.92 & 2.08 & 2.05 & 1.45 & 1.61 & 1.33 & 1.70 & 1.53 & 2.16 \\
\hline Cinnamic acid & 0.13 & 0.08 & 0.08 & 0.09 & 0.04 & 0.06 & 0.04 & 0.07 & 0.05 & 0.05 \\
\hline Pinobanksin & 0.32 & 0.43 & - & - & 0.45 & 0.35 & - & - & - & - \\
\hline Kaempferol & 0.21 & - & 0.21 & - & - & 0.15 & 0.16 & - & 0.17 & 0.29 \\
\hline Compounds & C 11 & C 12 & C 13 & C 14 & C 15 & C 16 & C 17 & C 18 & C 19 & C 20 \\
\hline p-Coumaric acid & 0.01 & 0.18 & 0.29 & 0.14 & 0.21 & 0.35 & 0.19 & 0.68 & 0.14 & 0.01 \\
\hline Lindenin & 17.03 & 21.00 & 38.62 & 19.79 & 16.18 & 35.79 & 17.82 & 23.11 & 17.56 & 81.03 \\
\hline trans, trans -abscisic acid & 0.70 & 0.13 & 0.29 & 0.33 & 0.75 & 0.72 & 0.25 & 0.98 & 0.23 & 1.66 \\
\hline cis, trans-abscisic acid & 2.44 & 6.21 & 1.65 & 1.58 & 3.38 & 2.24 & 1.05 & 3.14 & 1.17 & 5.38 \\
\hline Cinnamic acid & - & - & 一 & 0.04 & 一 & 0.09 & 0.03 & 0.12 & 0.07 & 0.17 \\
\hline Pinobanksin & - & - & 一 & - & - & - & - & - & - & - \\
\hline Kaempferol & 0.33 & - & 0.28 & - & - & - & 0.15 & - & 0.17 & 0.56 \\
\hline
\end{tabular}




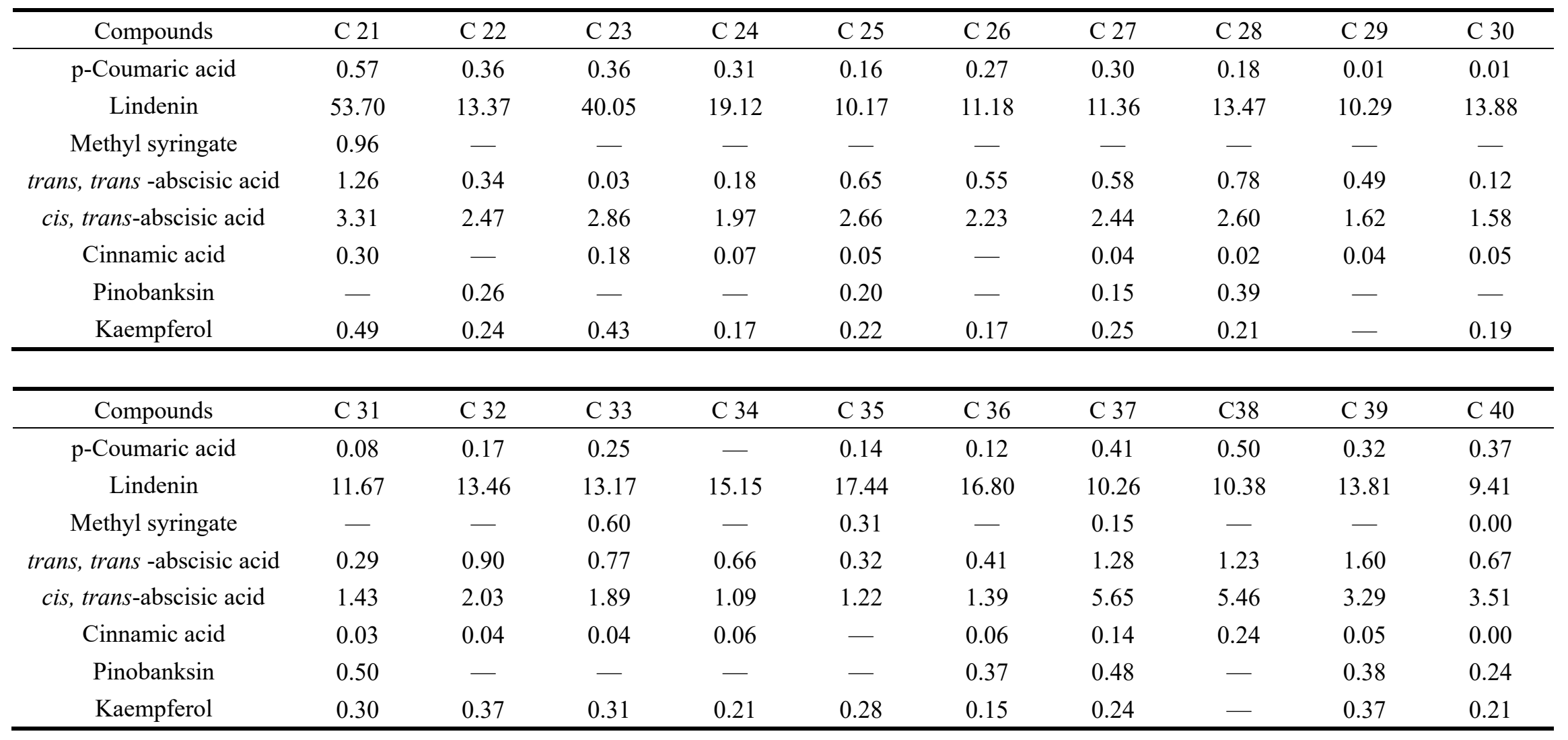

Note. Average calculated from two independent analyses. (一): not detected. 

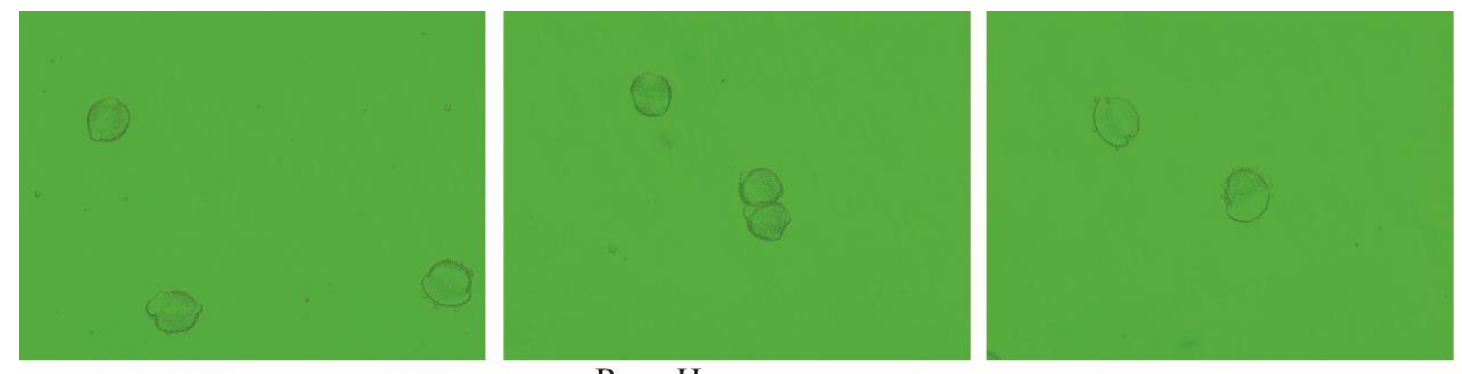

Rape Honey
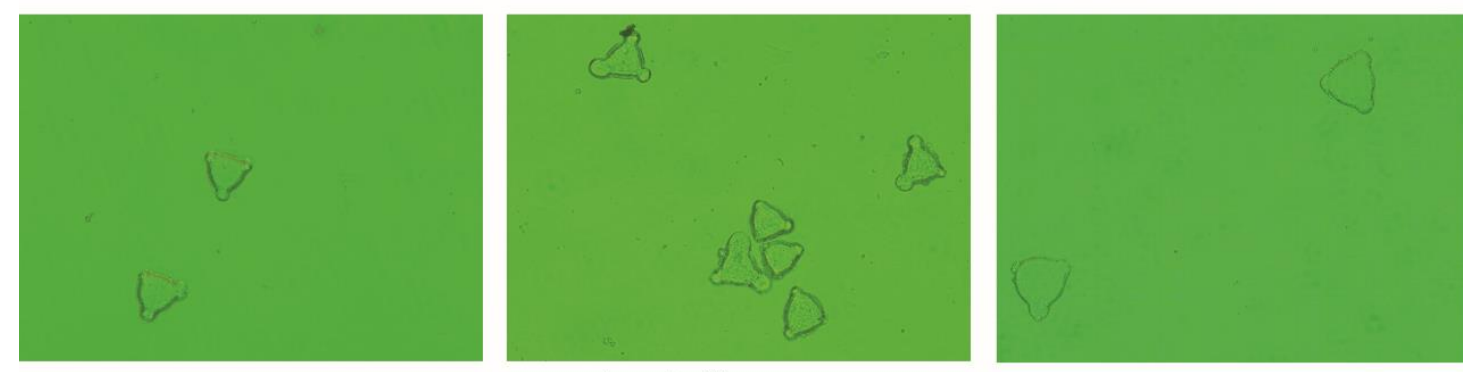

Acacia Honey
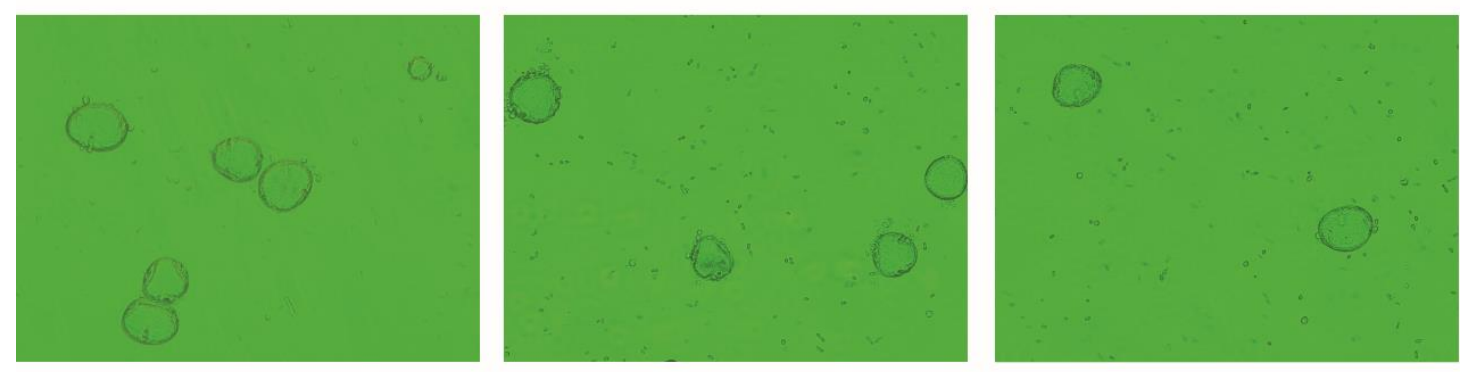

Linden Honey

Supplementary Figure 1. Pollen grain photomicrographs of monofloral honeys 

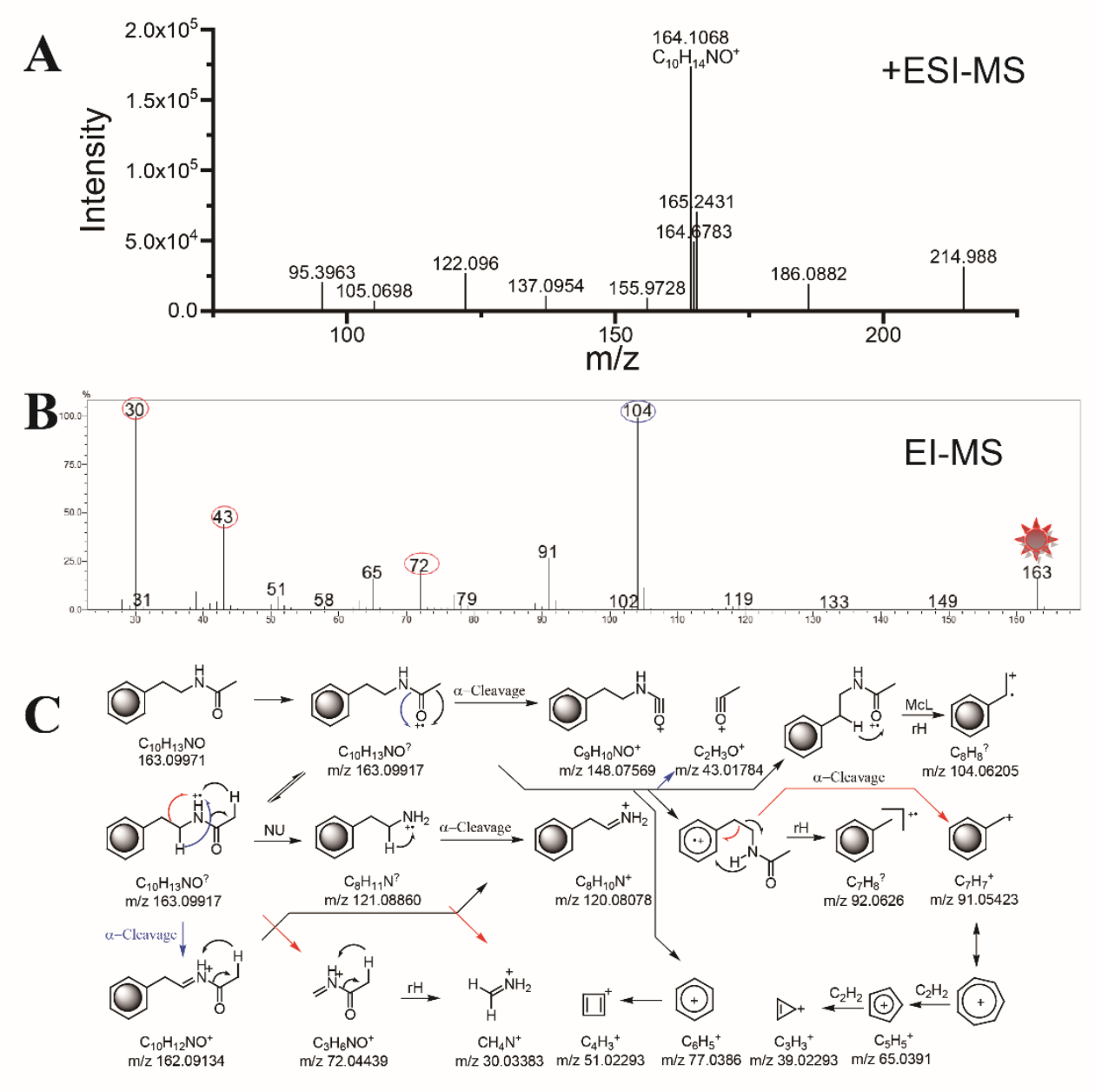

\section{Supplementary Figure 2. Mass spectra and fragment cleavage patterns of $\mathrm{N}$-(2- phenylethyl) acetamide}

Note: EI-MS of $N$-(2-phenylethyl) acetamide (A); fragment pathways of $N$-(2phenylethyl) acetamide in EI-MS (B). 

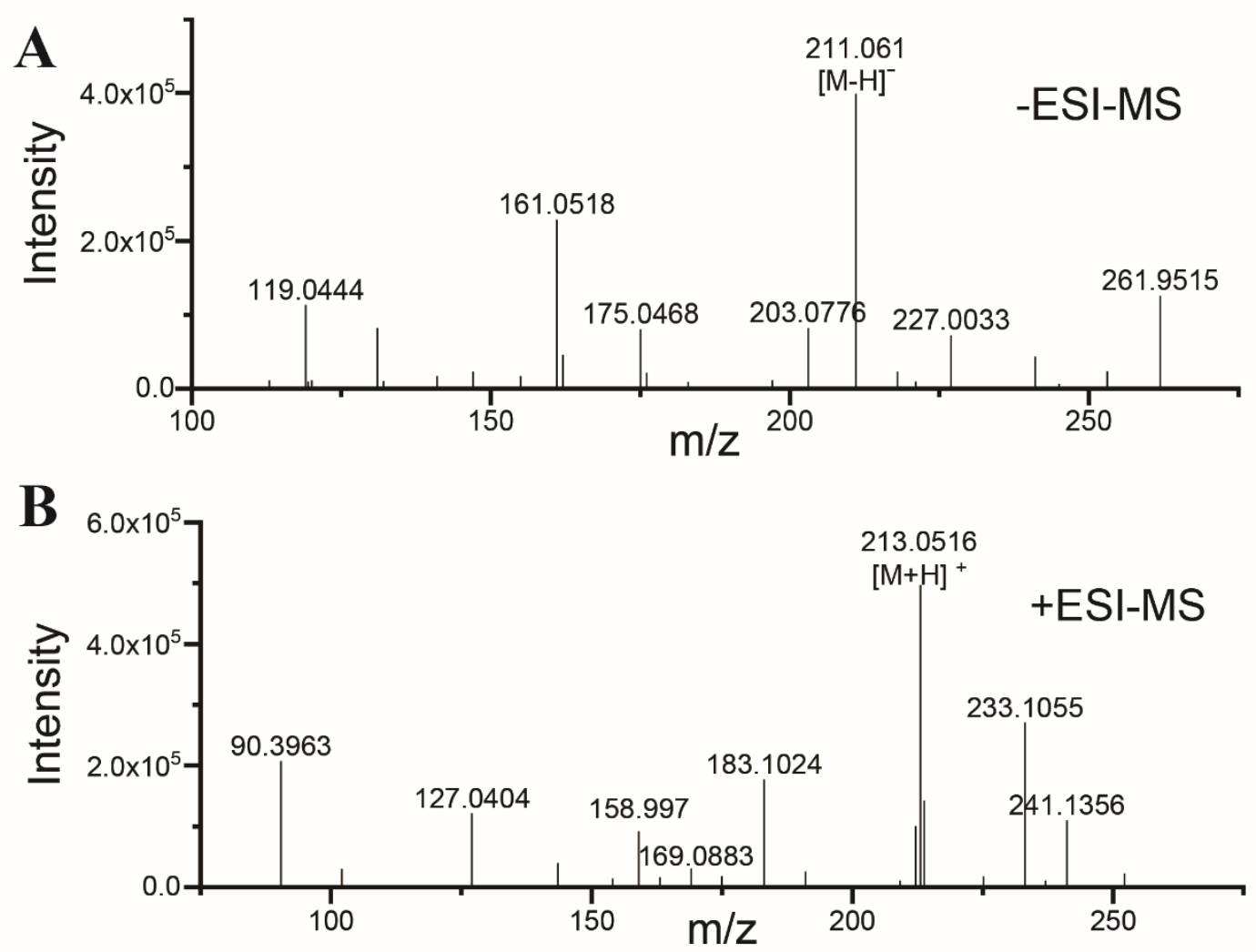

Supplementary Figure 3. Mass spectra of methyl syringate

Note: Negative ESI-MS of methyl syringate (A); positive ESI-MS of methyl syringate (B); 

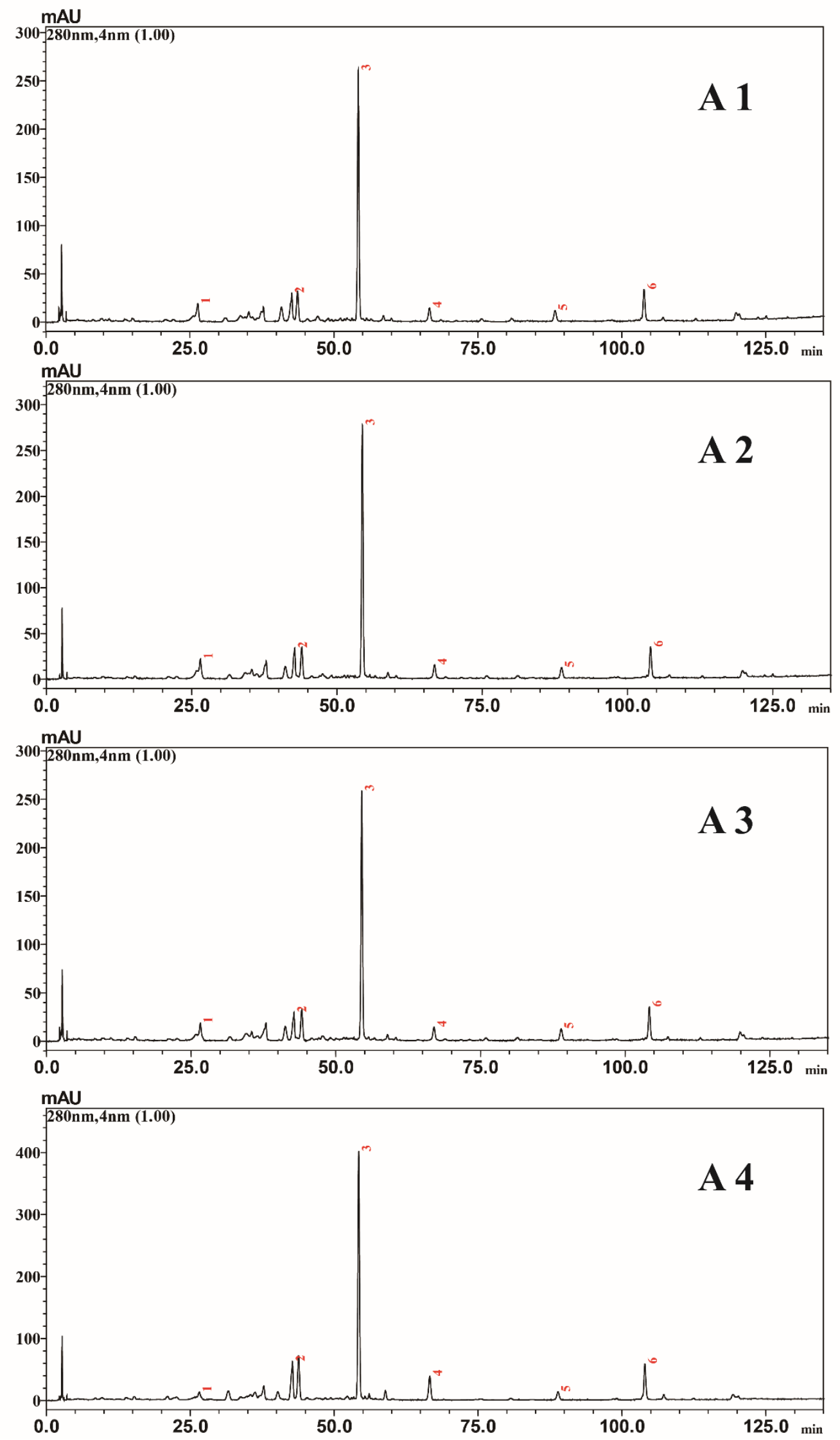

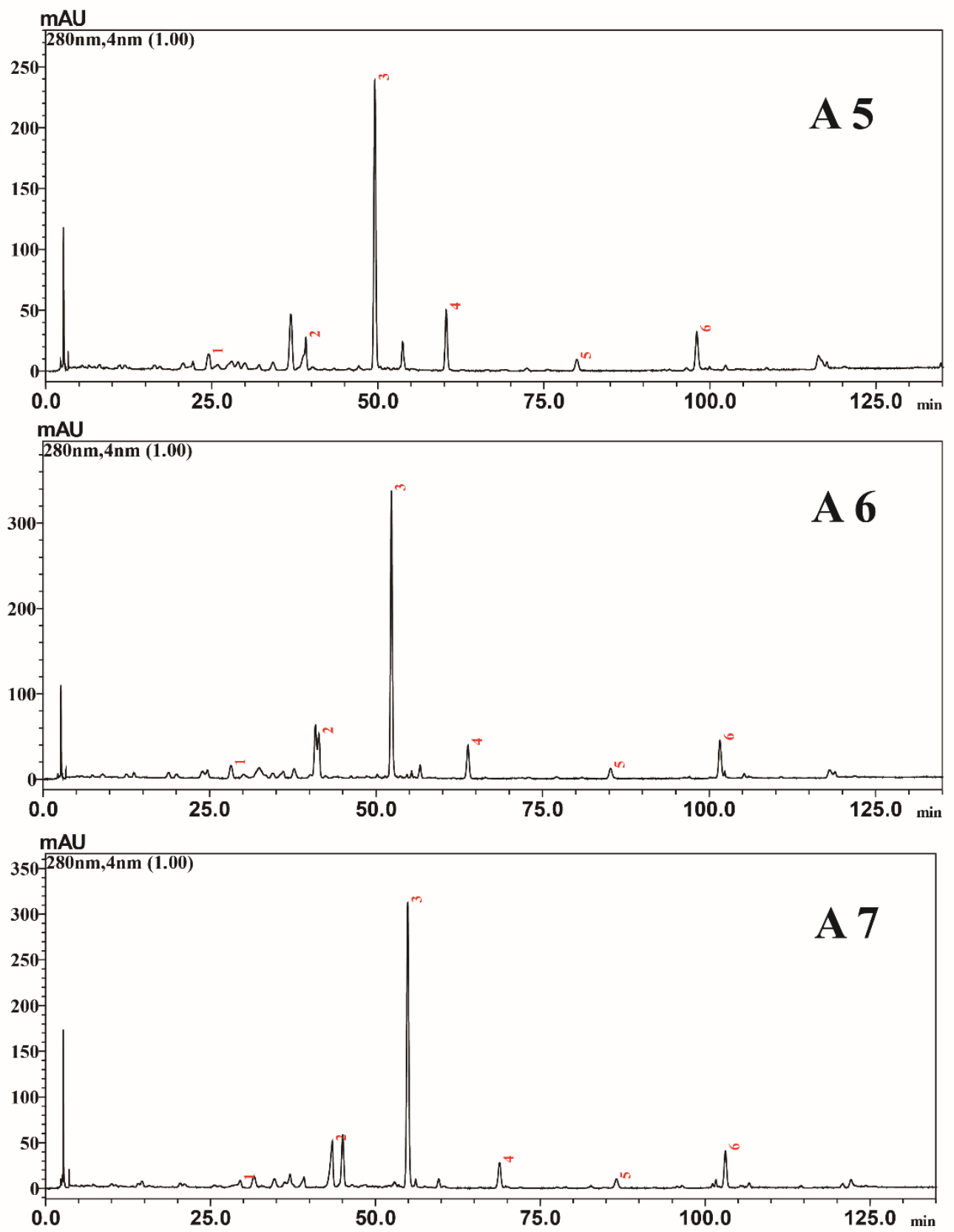

$$
\text { mAU }
$$

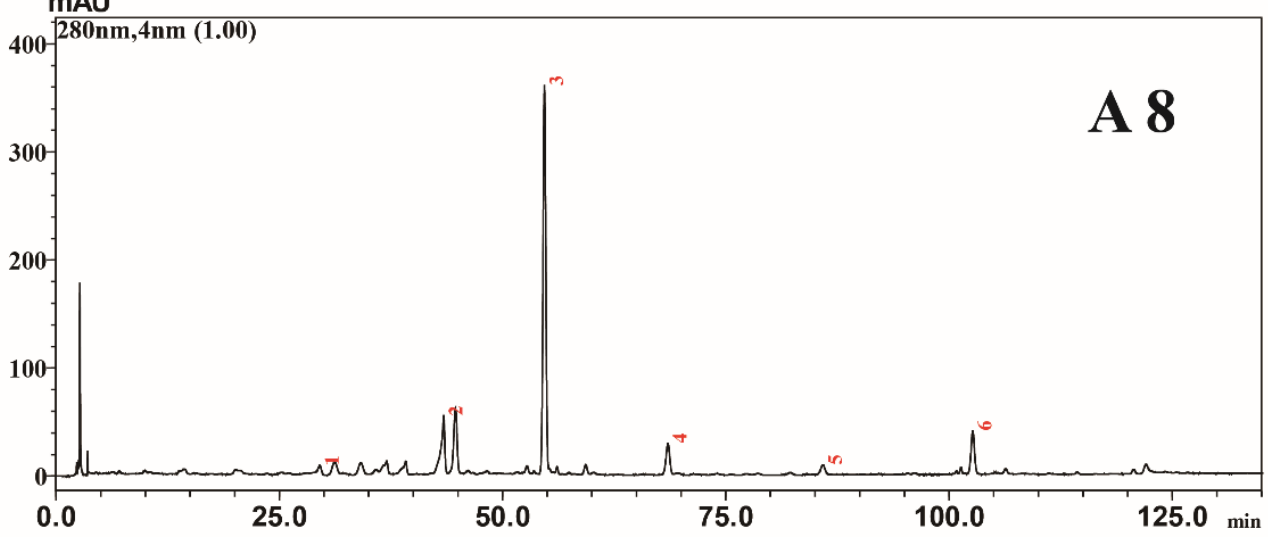



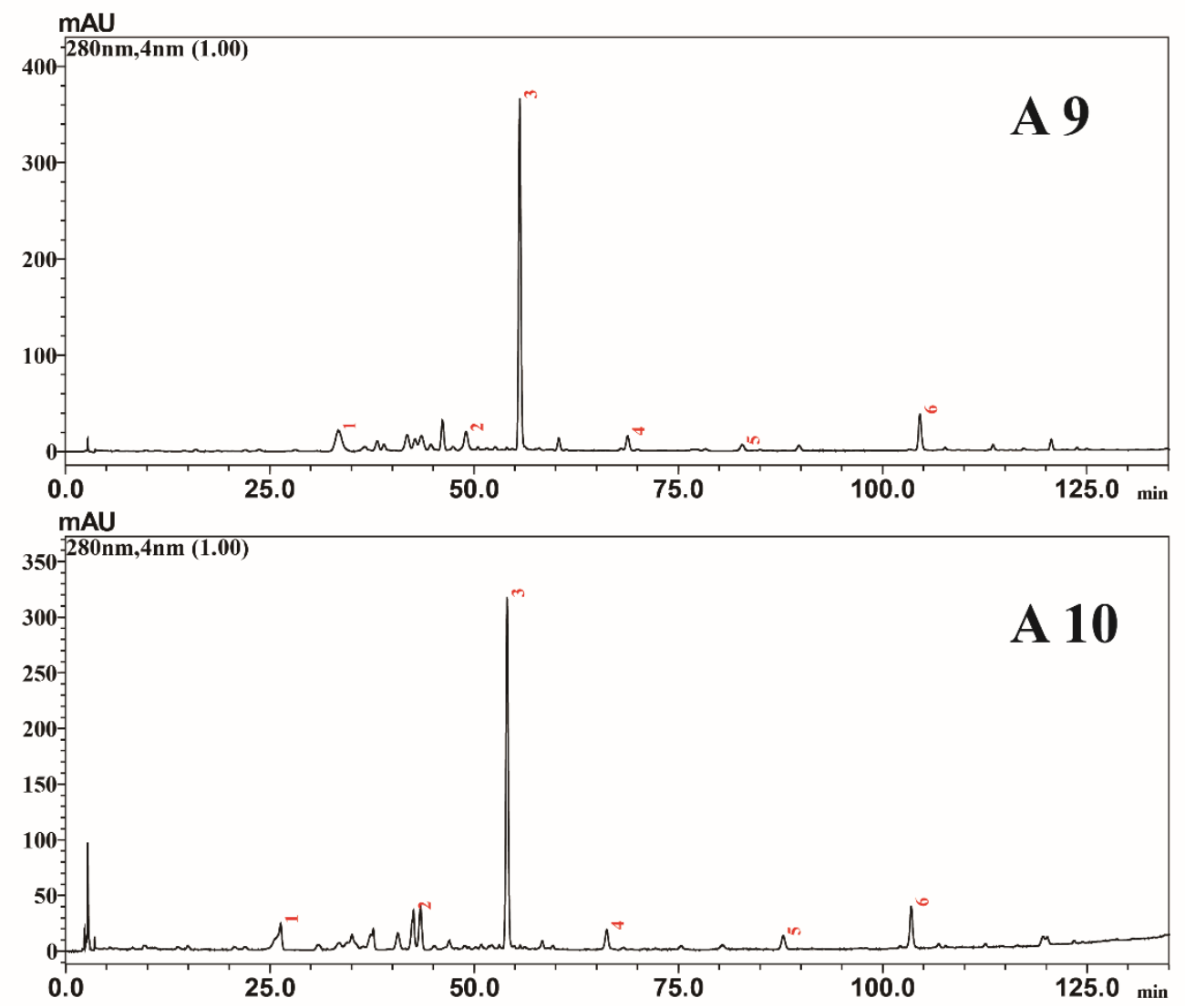

Supplementary Figure 4. HPLC profiles of raw rape honeys

Note: A1 to A10 represent sample 1 to 10. 

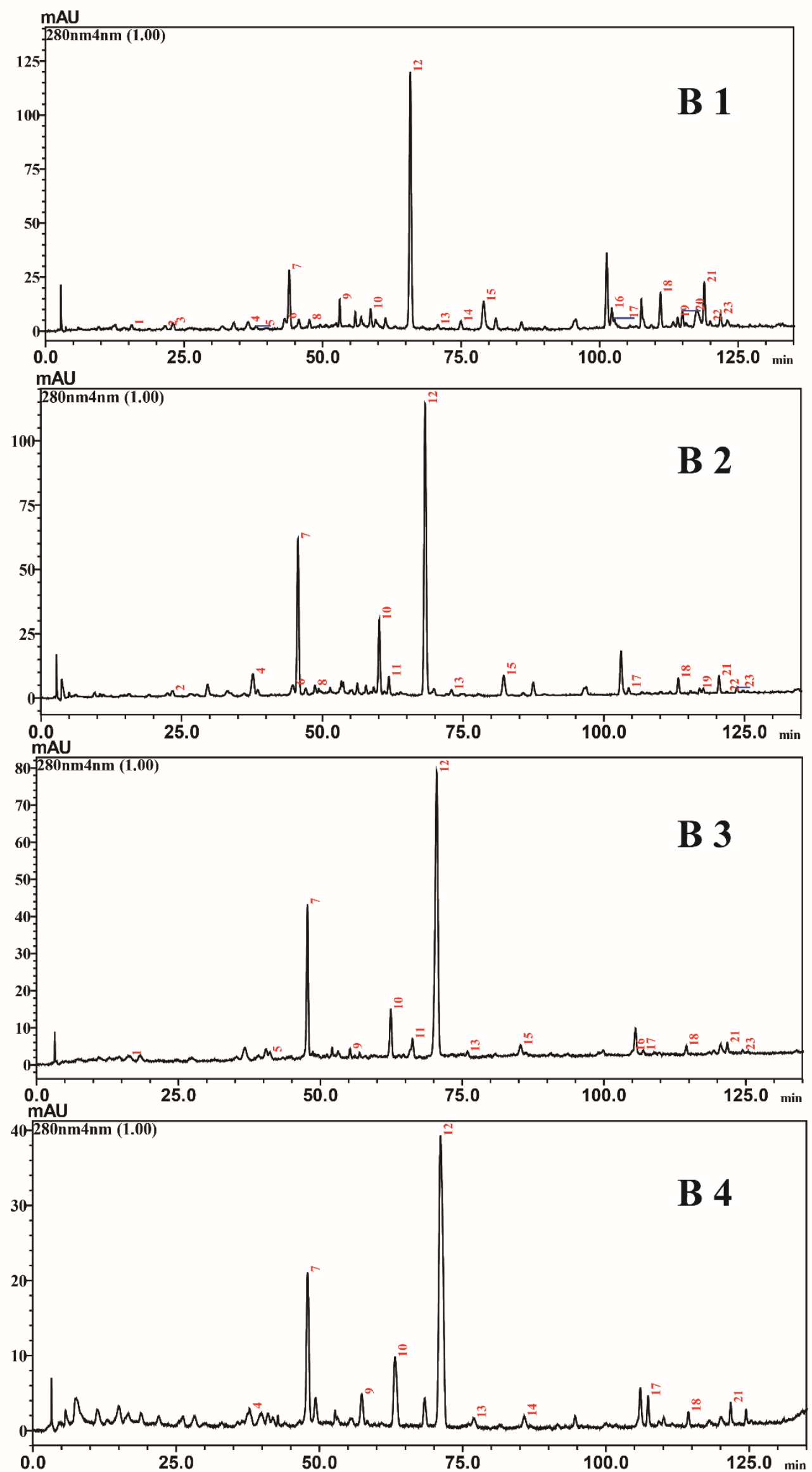

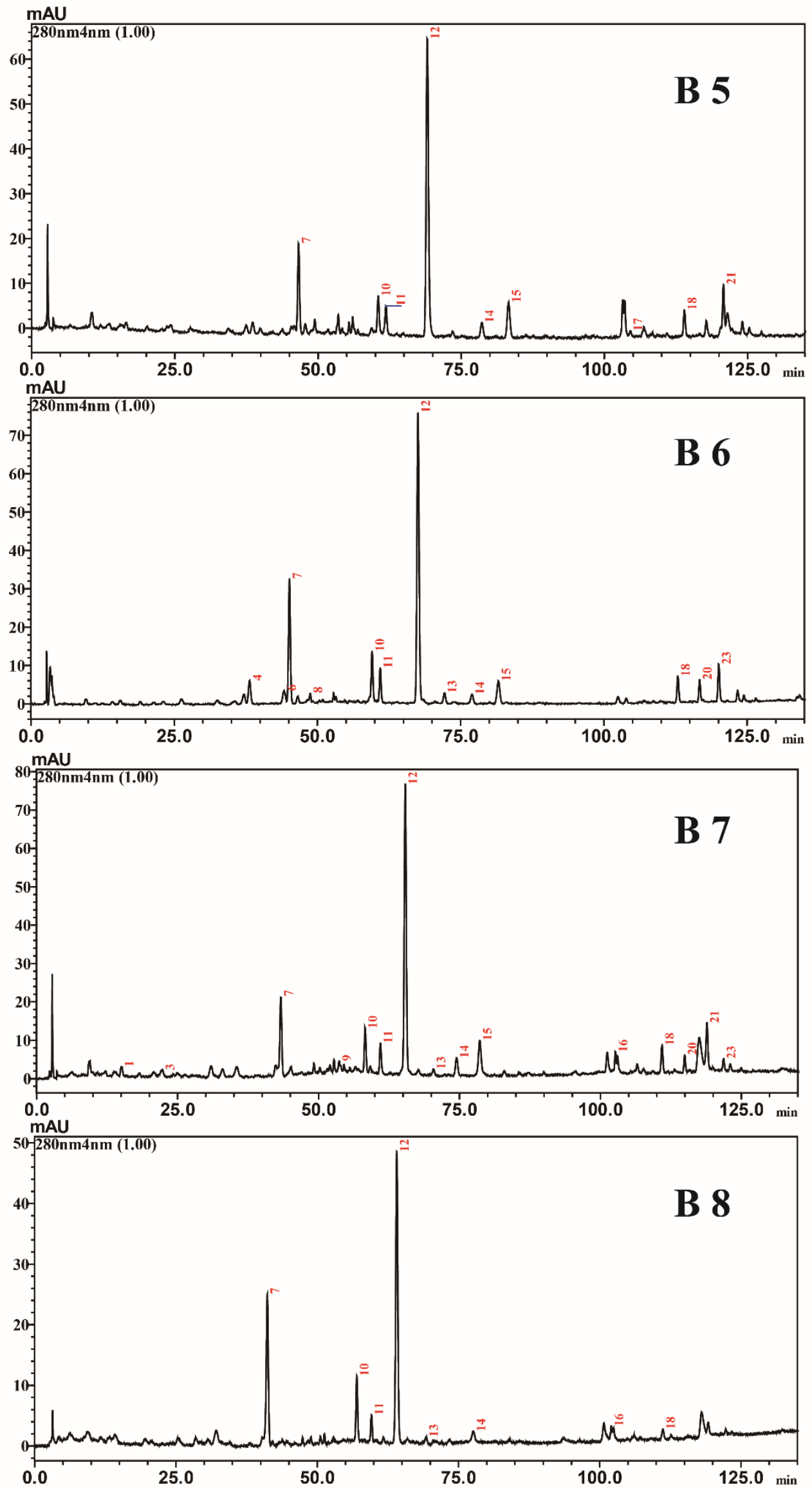

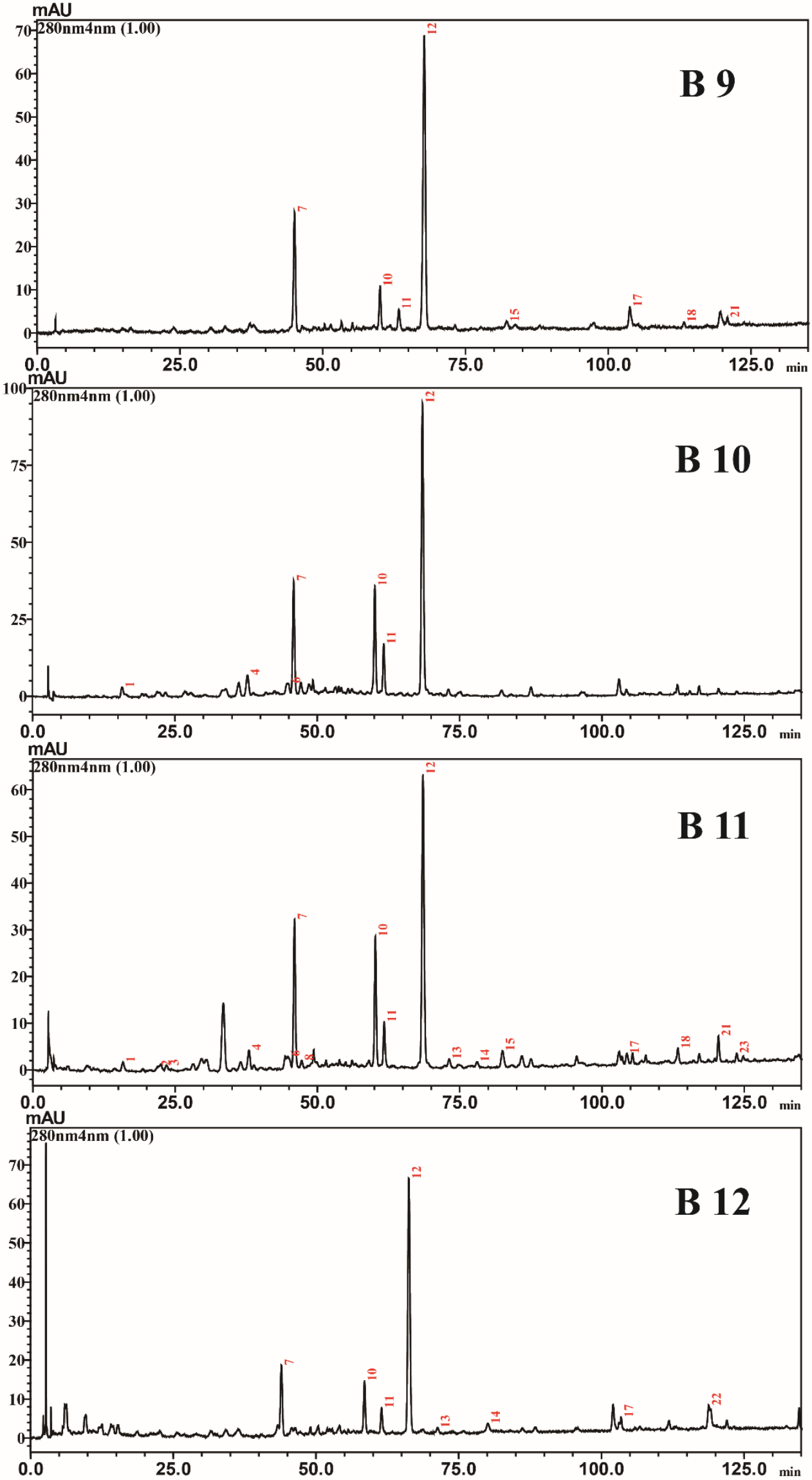

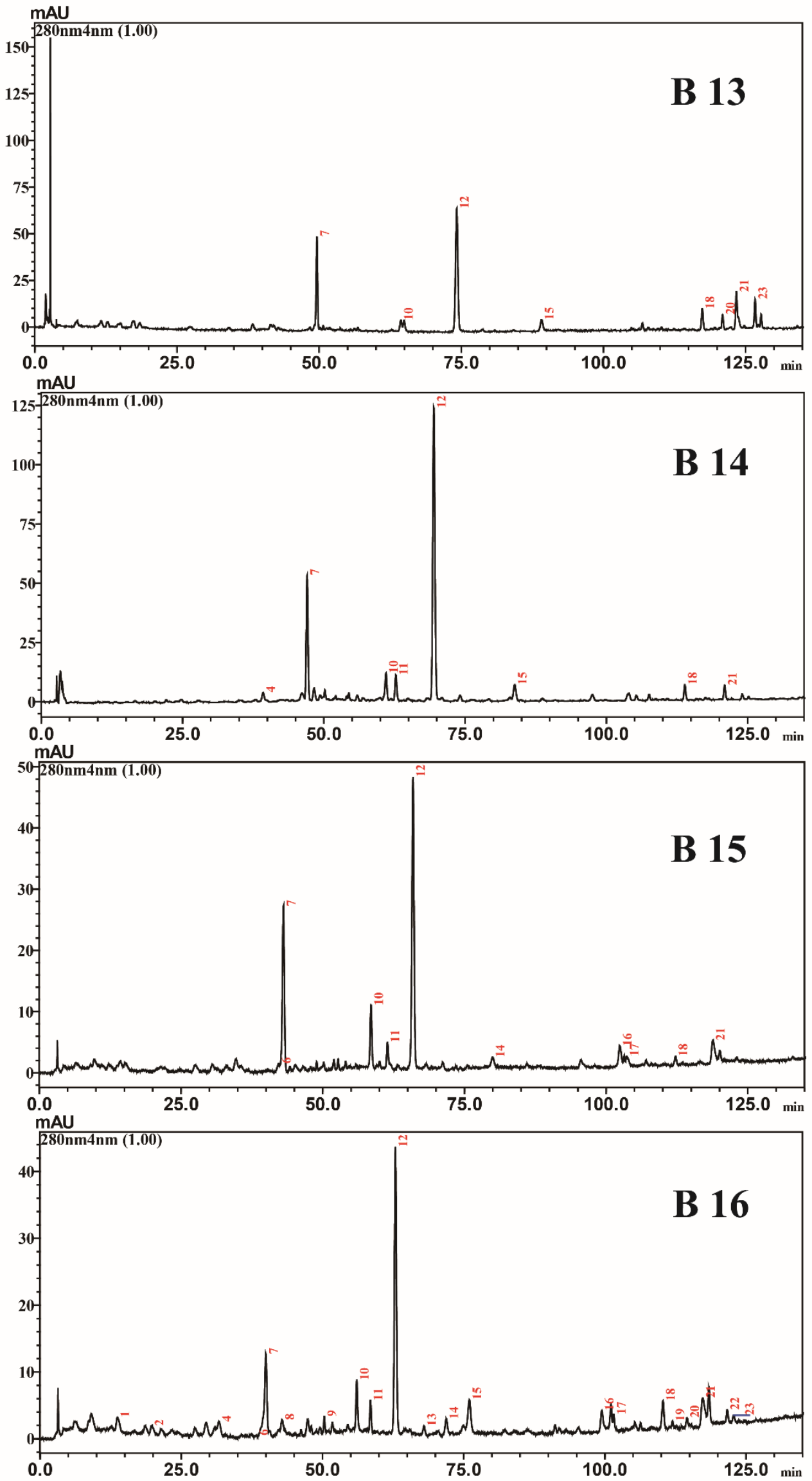

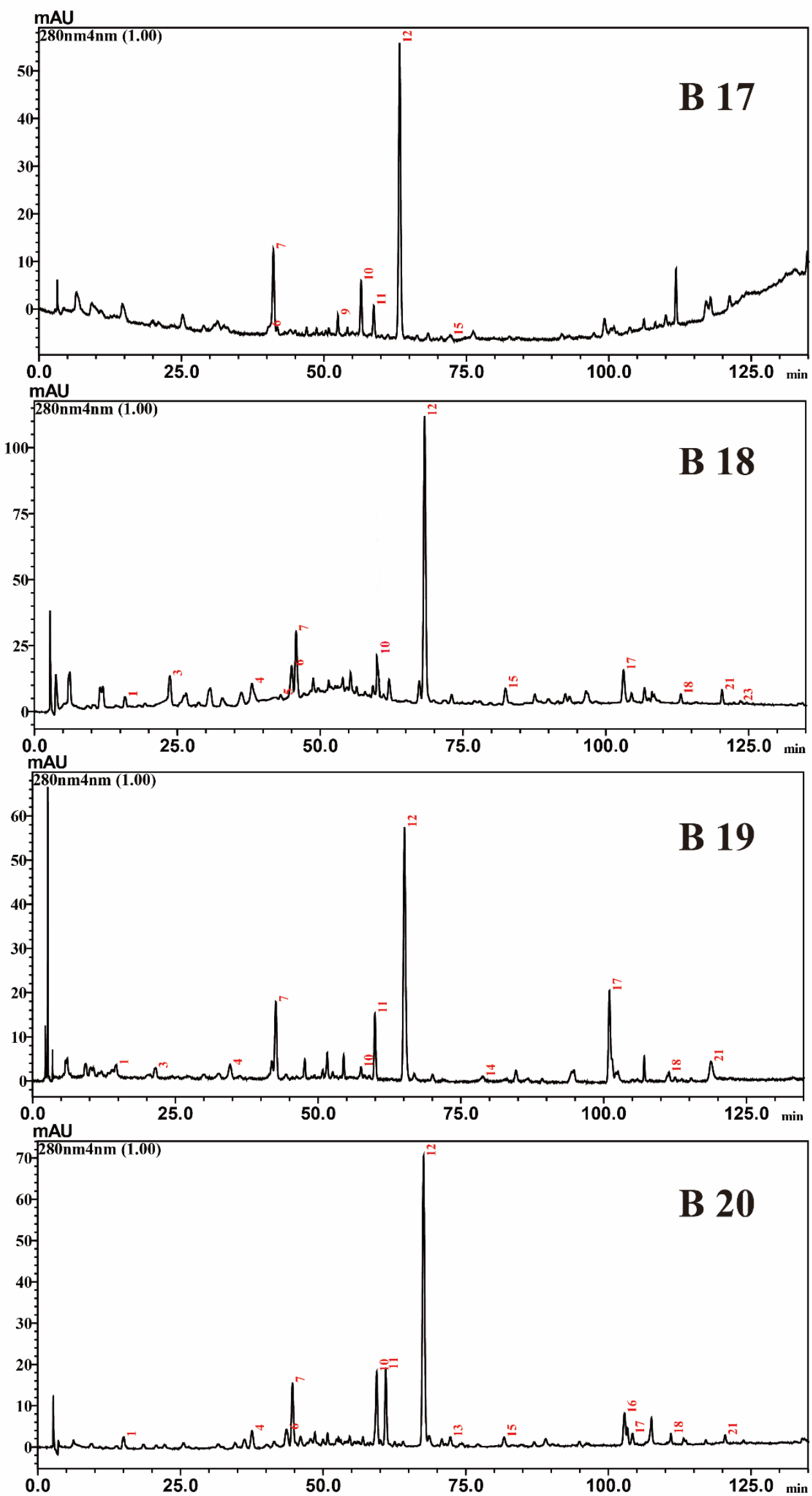

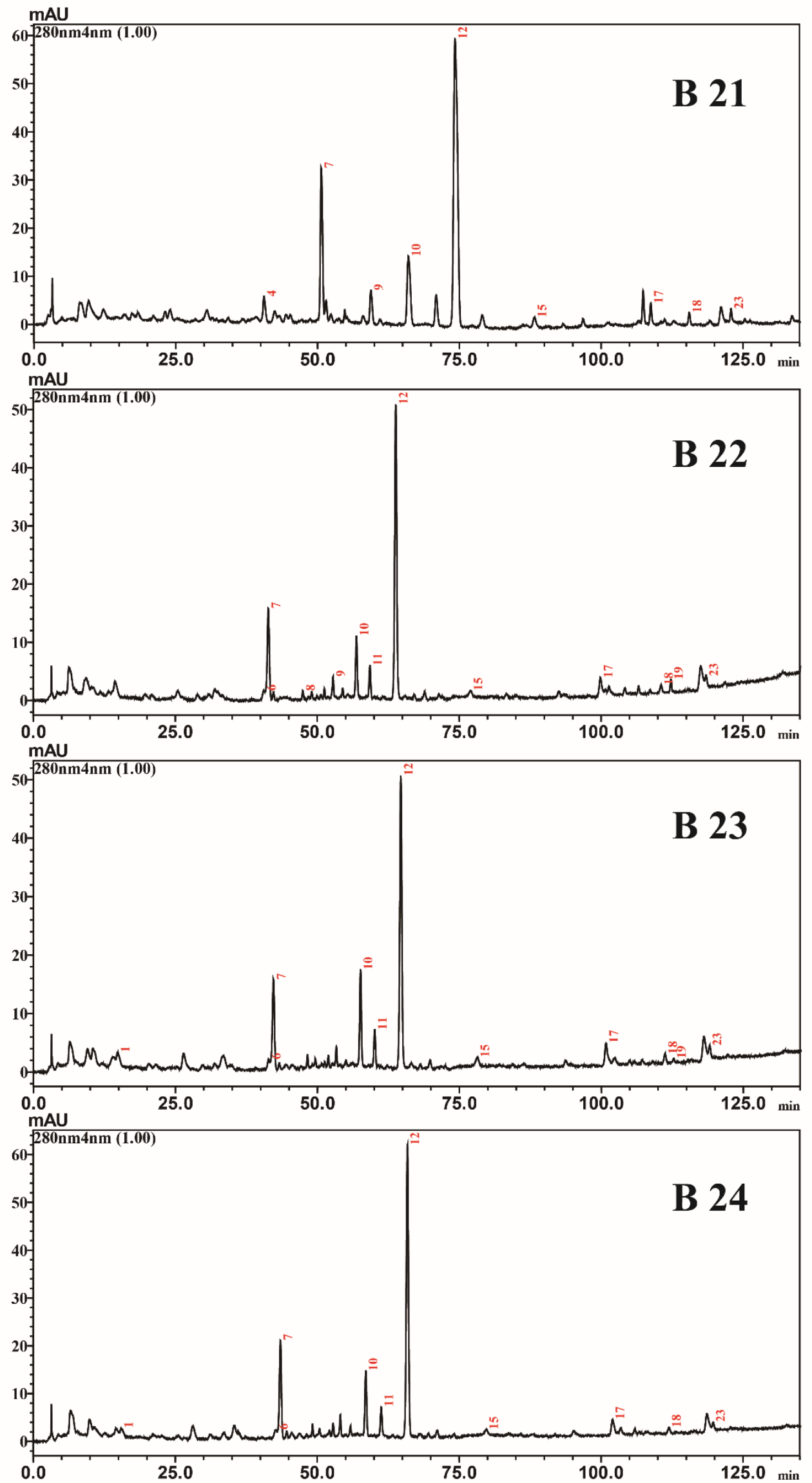


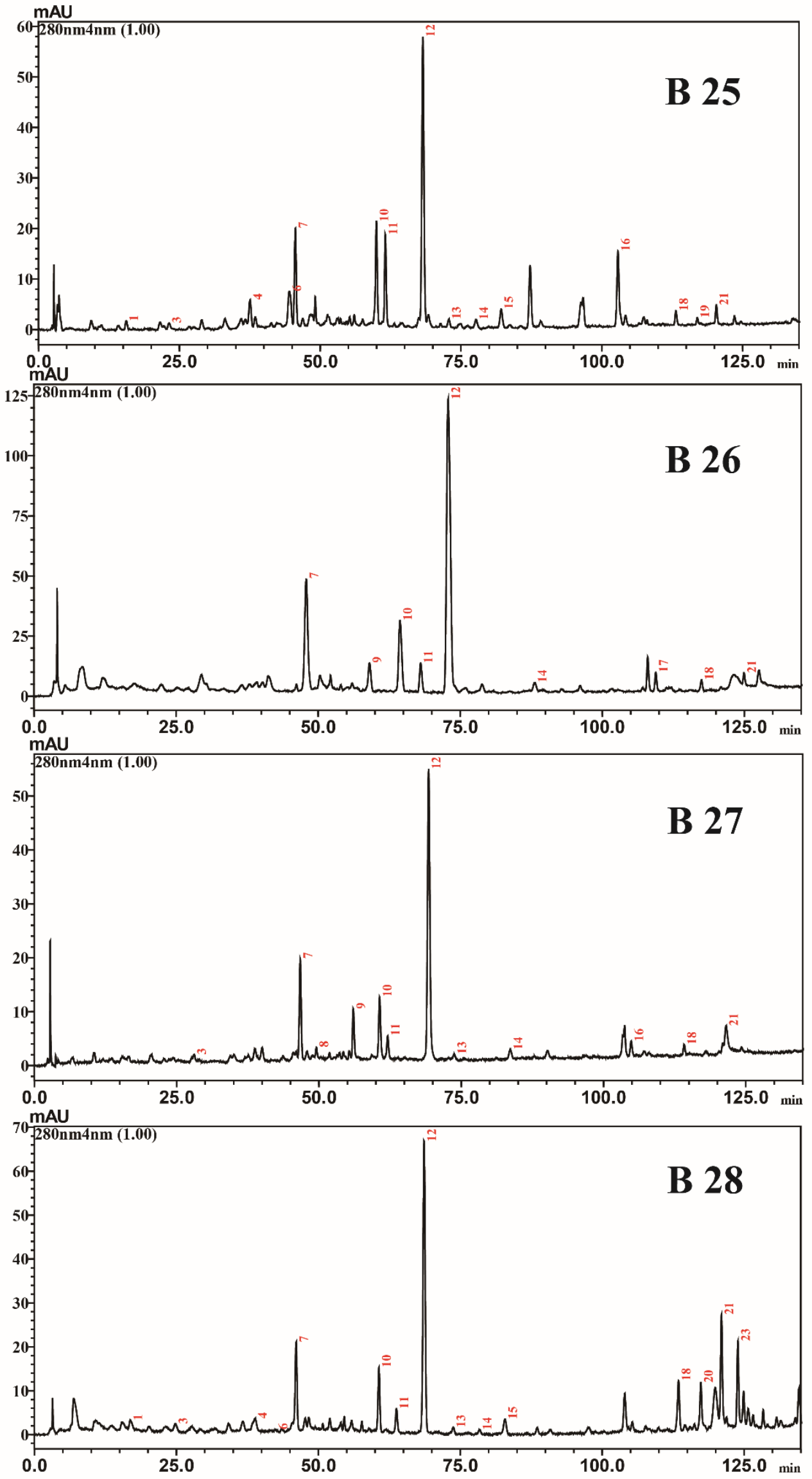



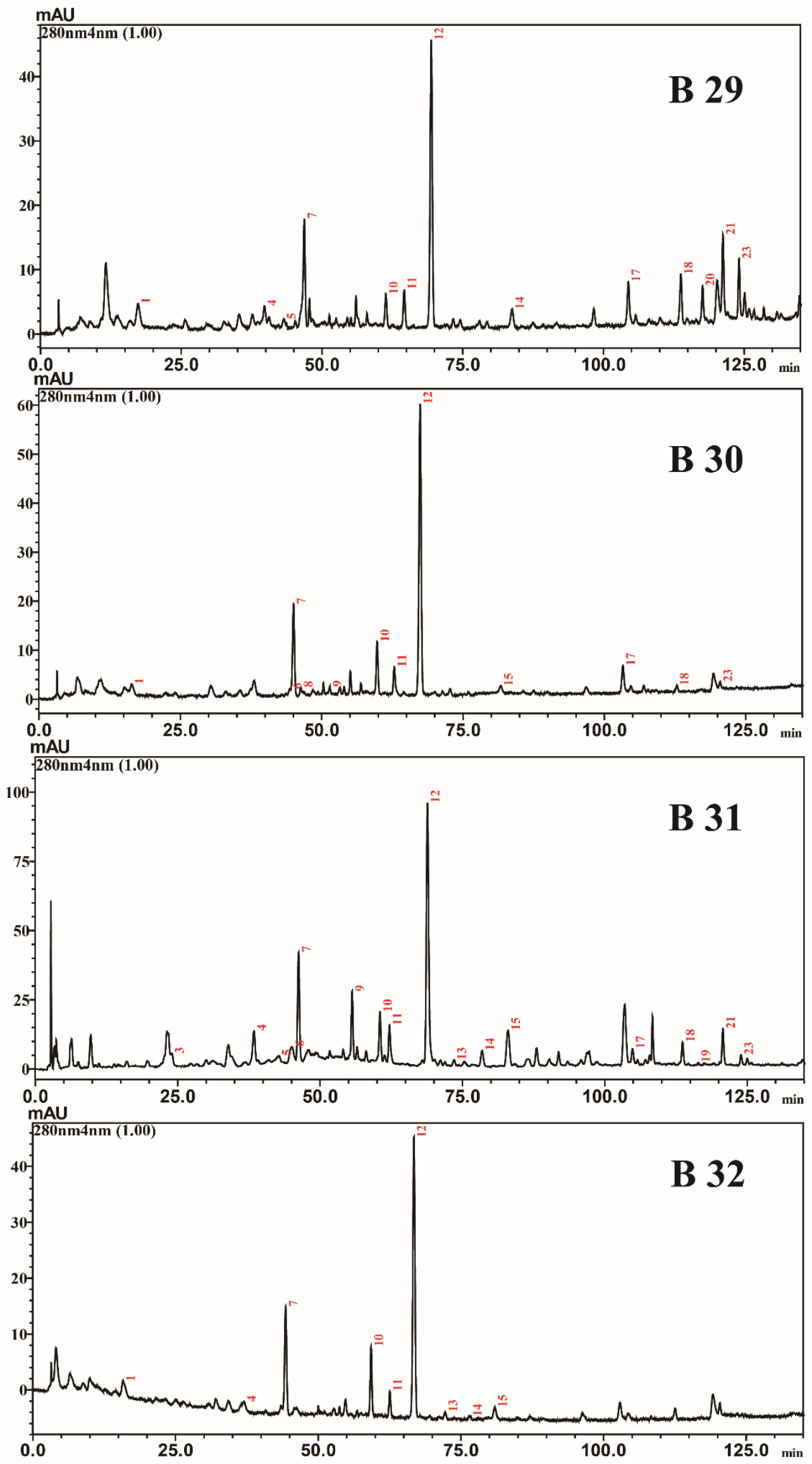

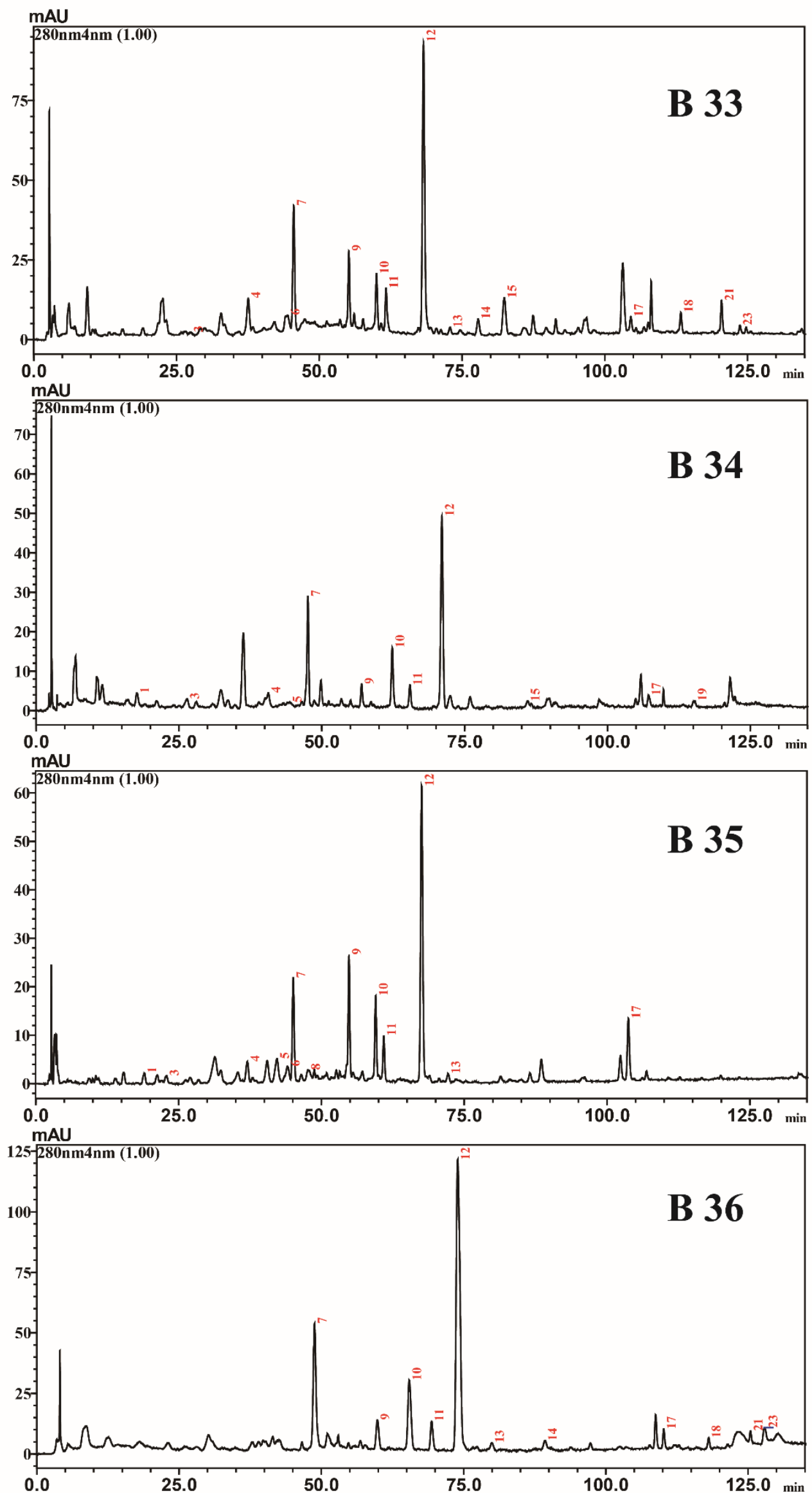

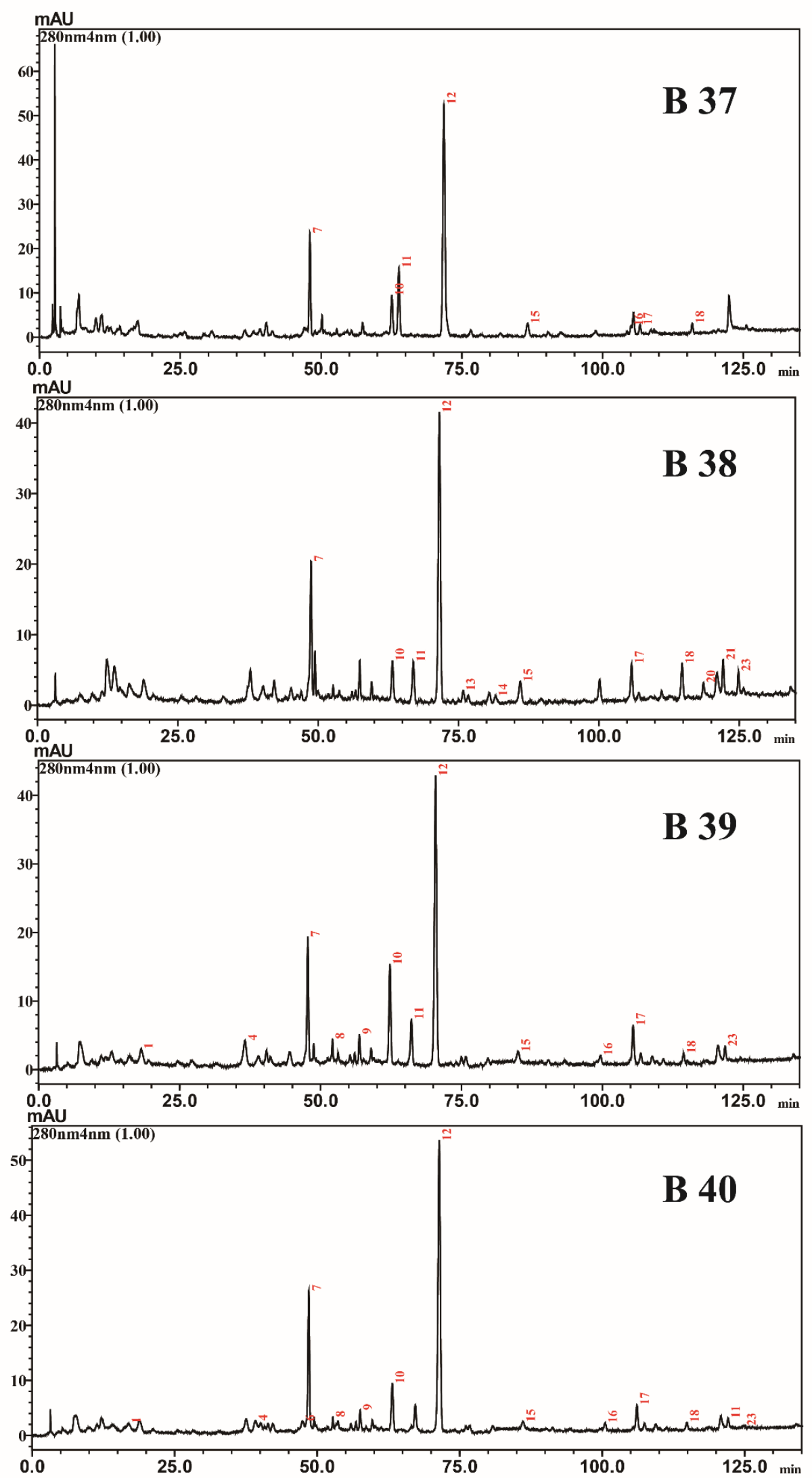

Supplementary Figure 5. HPLC profiles of raw acacia honeys

Note: B1 to B40 represent sample 1 to 40. 

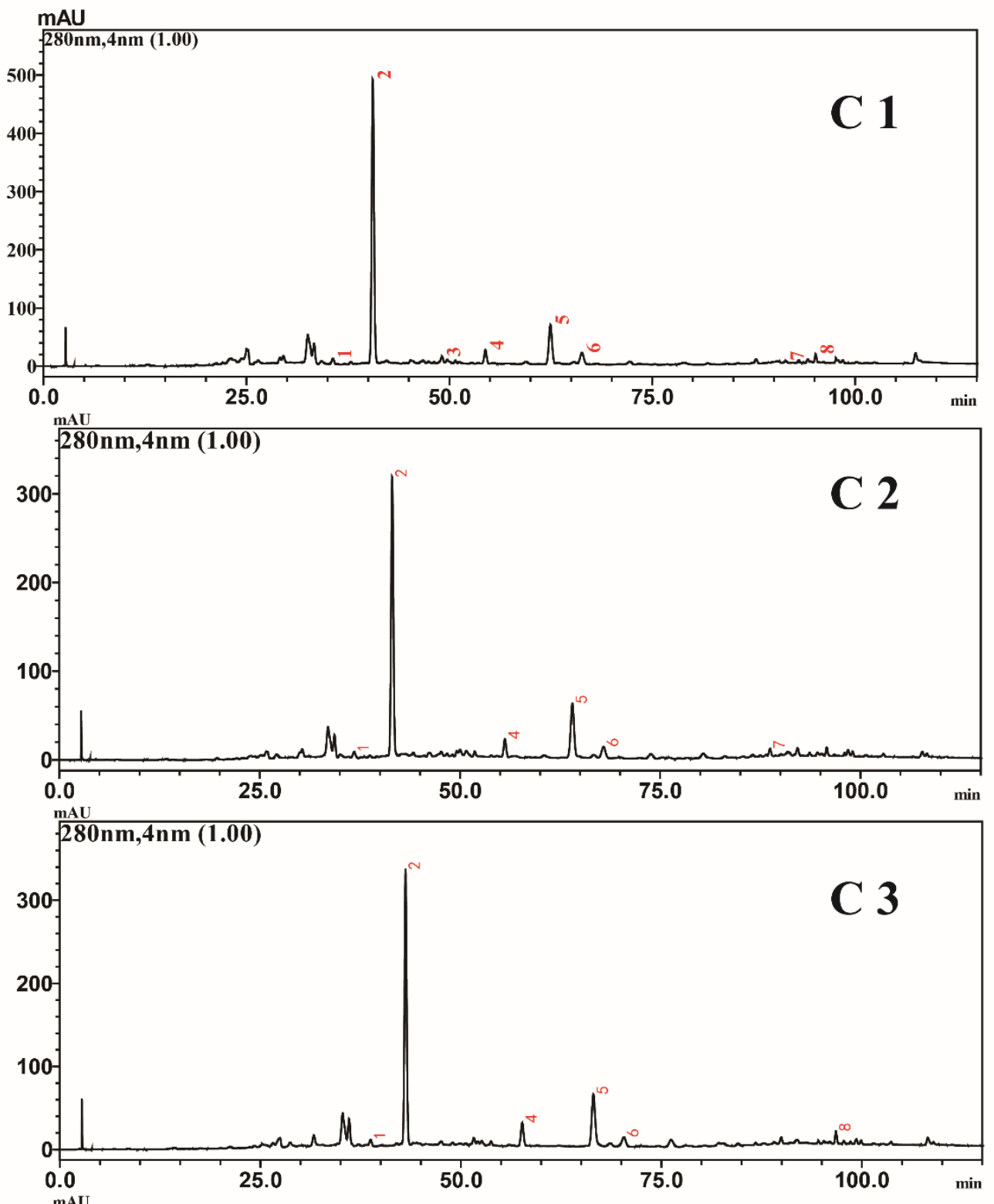

$280 \mathrm{~nm}, 4 \mathrm{~nm}$ (1.00)

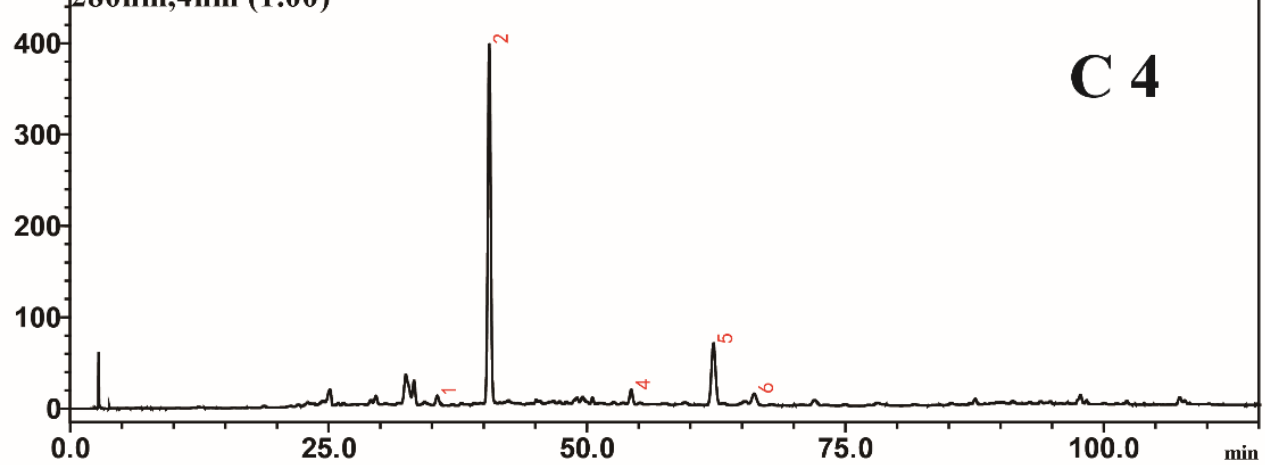




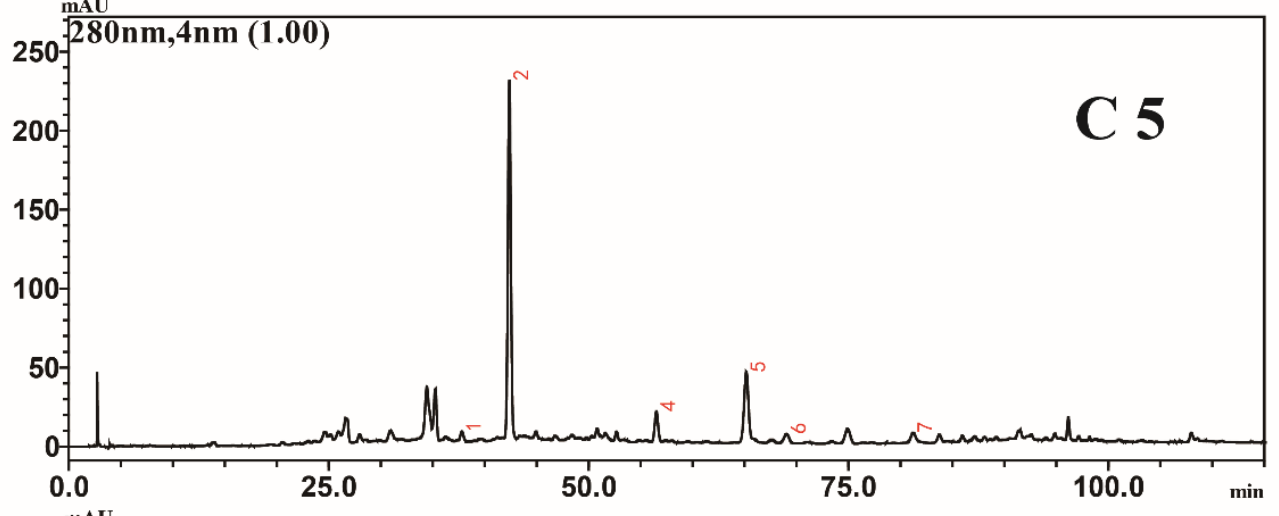

$250-\frac{\mathrm{mAU}}{280 \mathrm{~nm}, 4 \mathrm{~nm}(1.00)}$
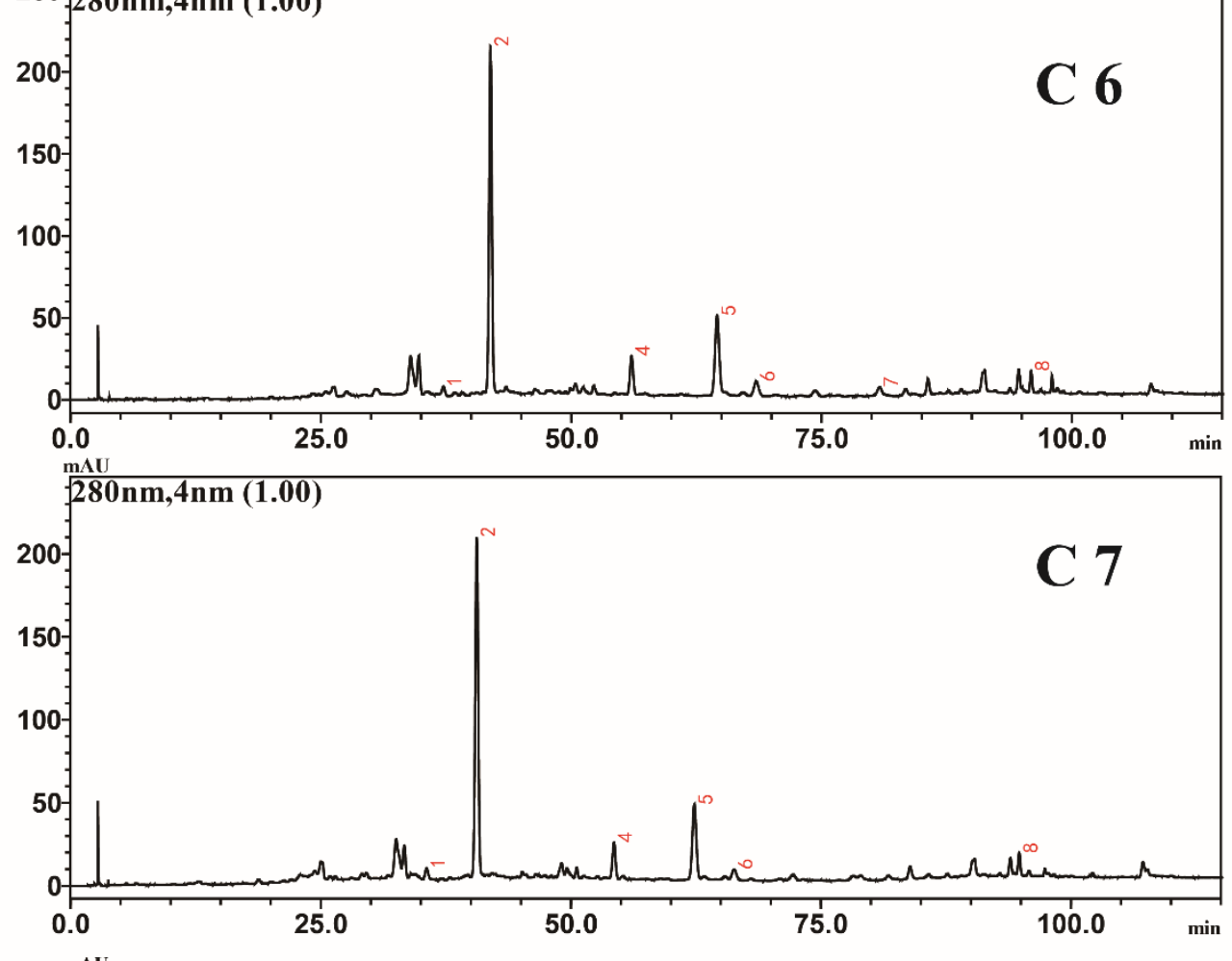

280nm,4nm (1.00)

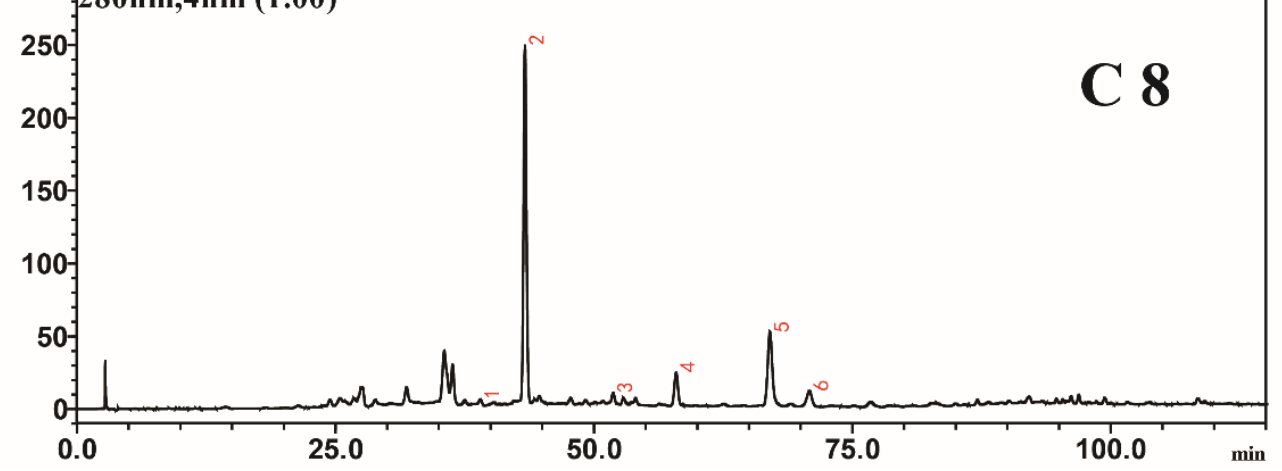



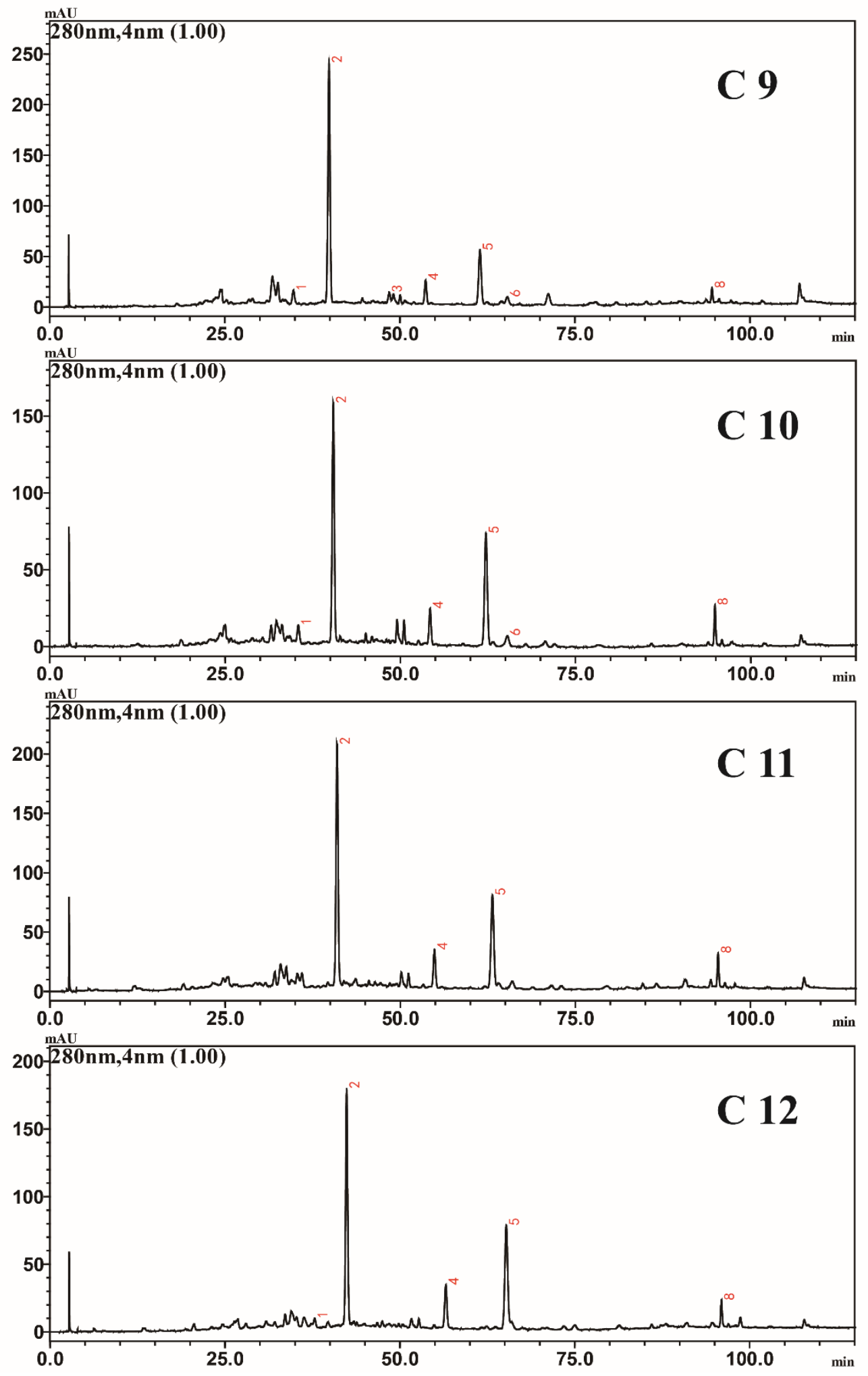
280nm,4nm (1.00)

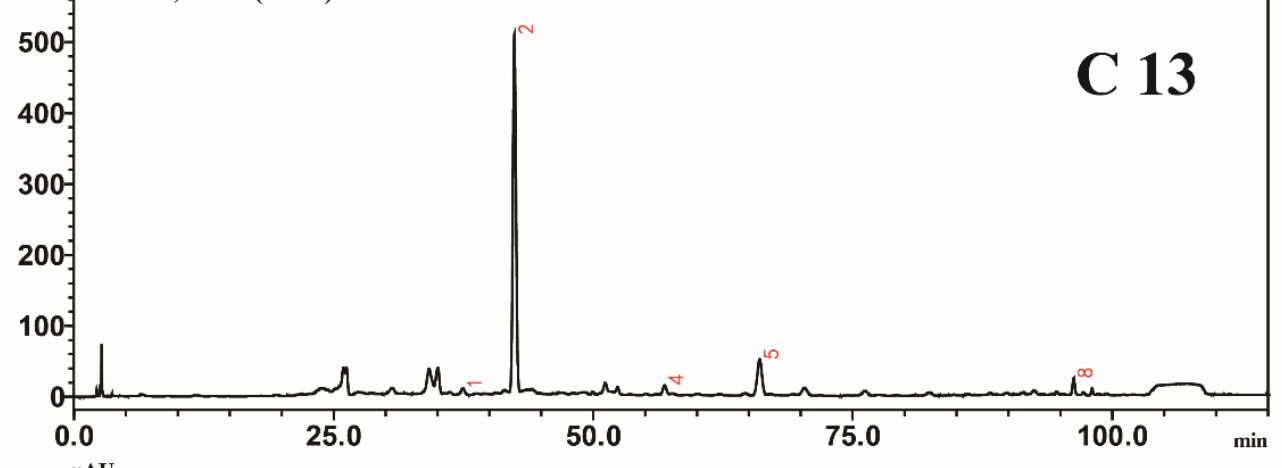

$250-280 \mathrm{~nm}, 4 \mathrm{~nm}$ (1.00)
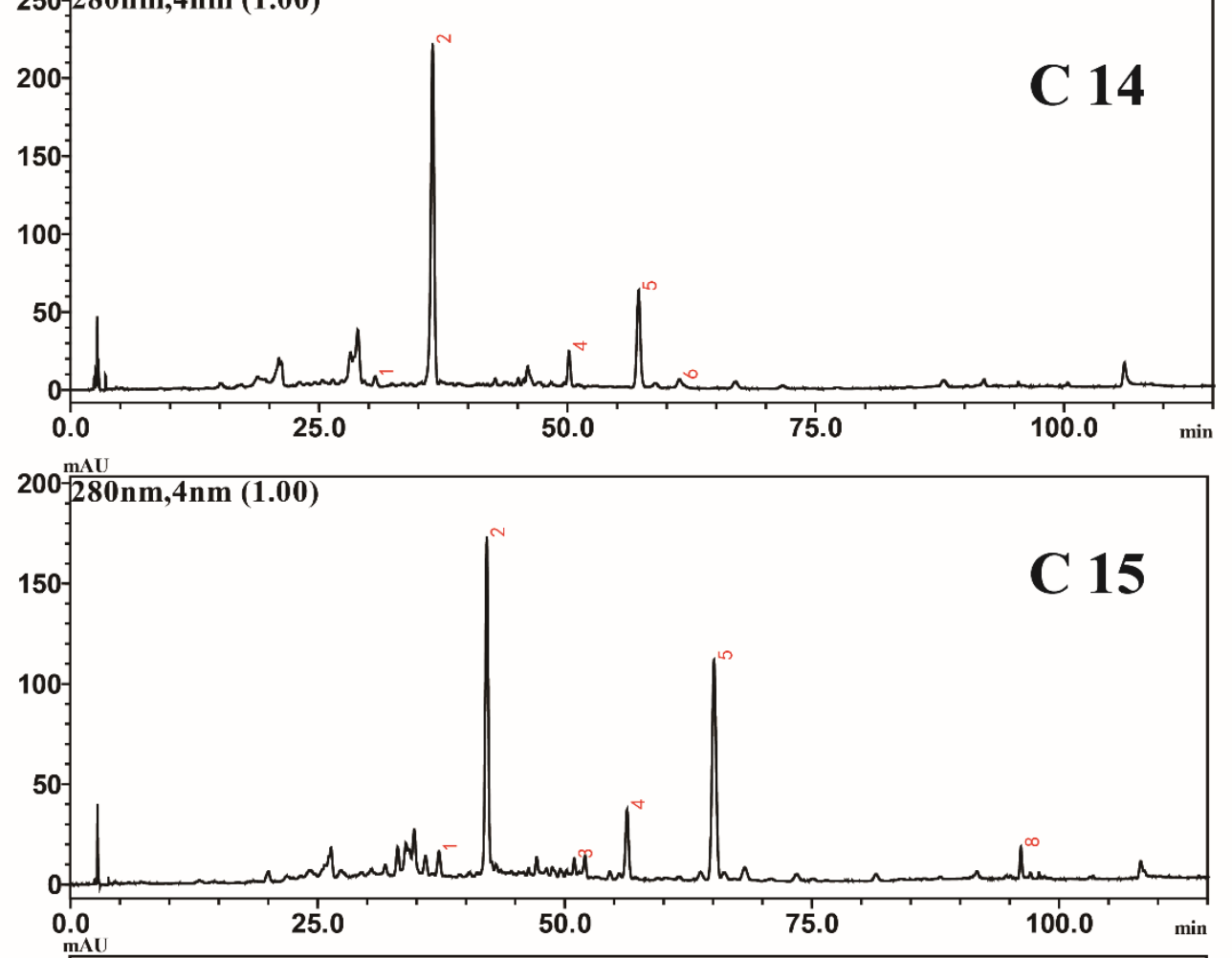

$280 \mathrm{~nm}, 4 \mathrm{~nm}(1.00)$

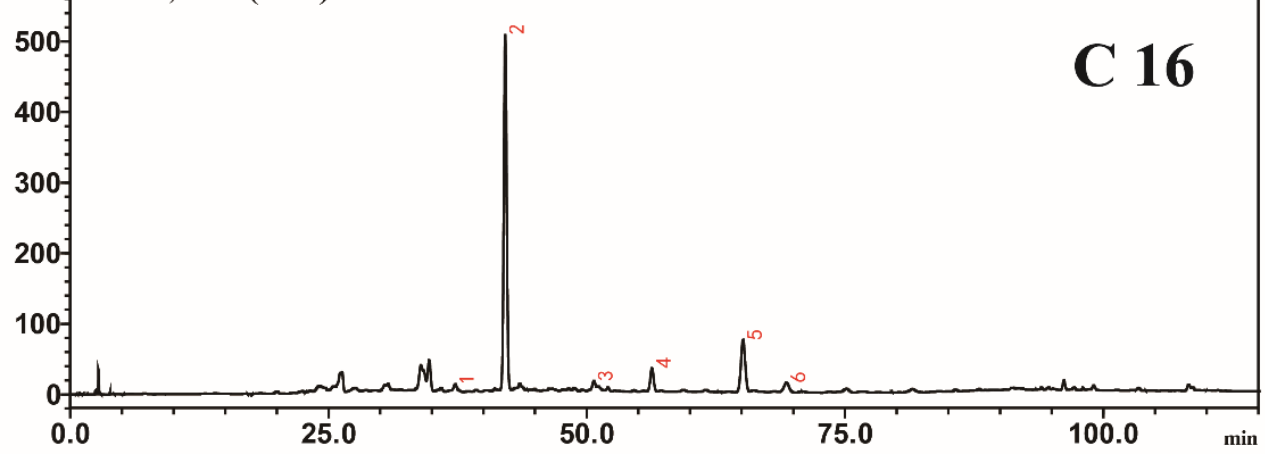




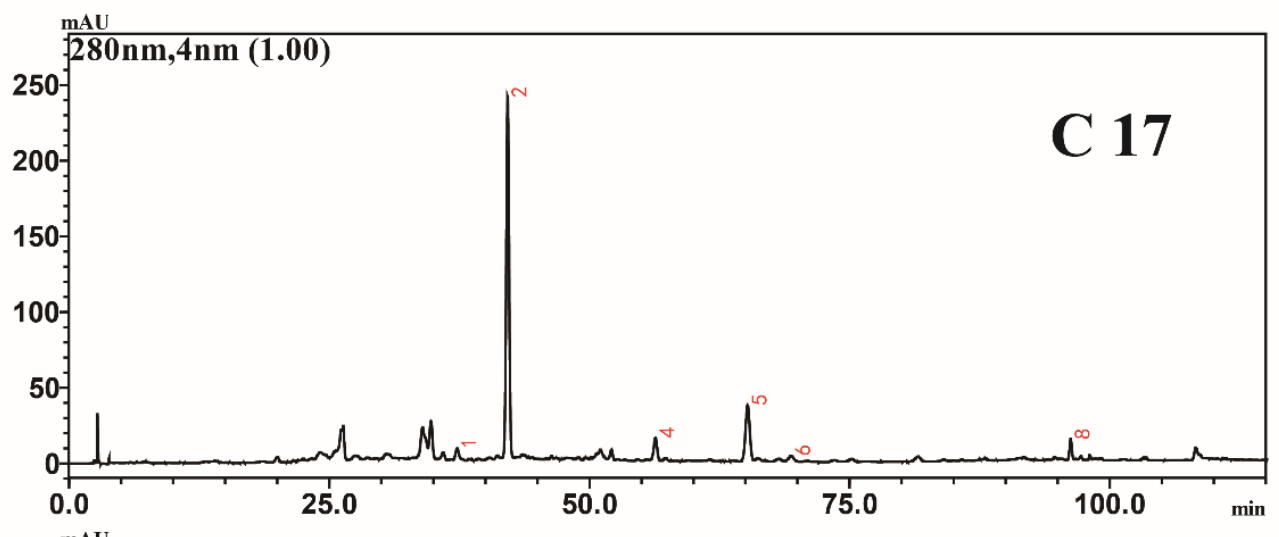

$250-\frac{\mathrm{maU}}{280 \mathrm{~nm}, 4 \mathrm{~nm}(1.00)}$

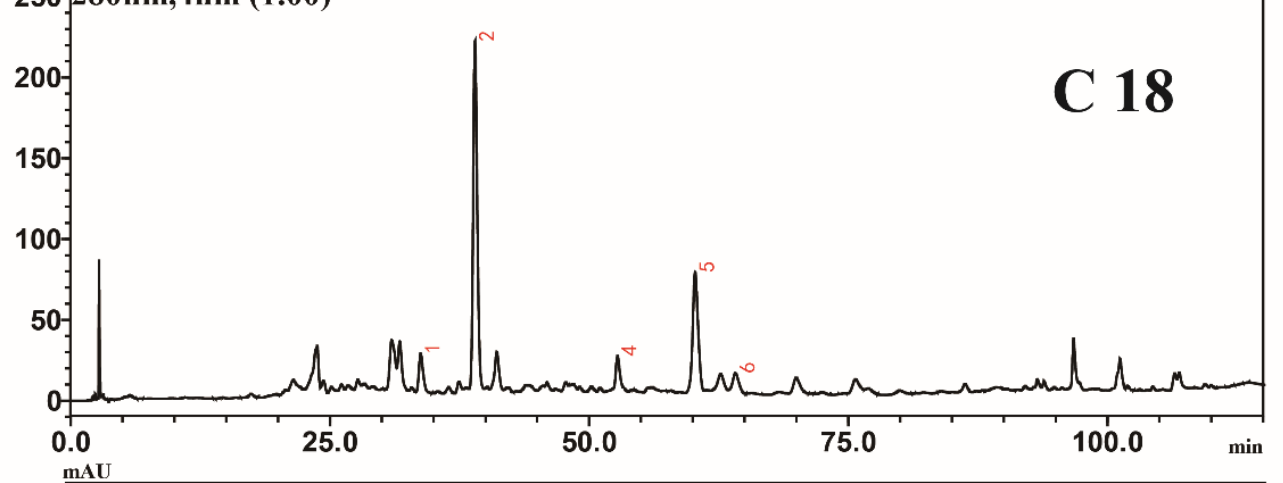

mAU

50.0

$200-280 \mathrm{~nm}, 4 \mathrm{~nm}$ (1.00)

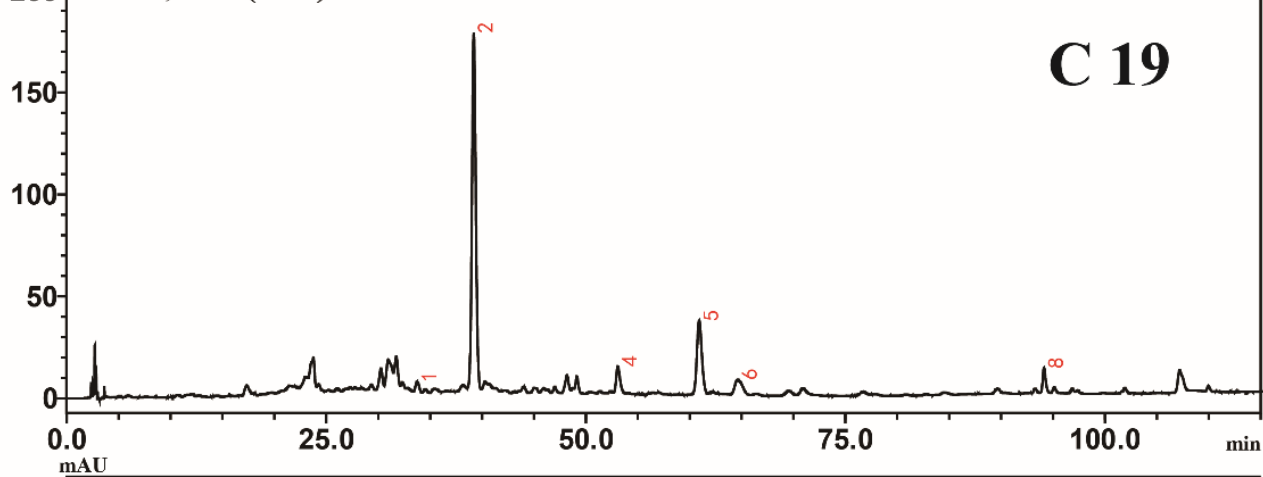

$280 \mathrm{~nm}, 4 \mathrm{~nm}$ (1.00)

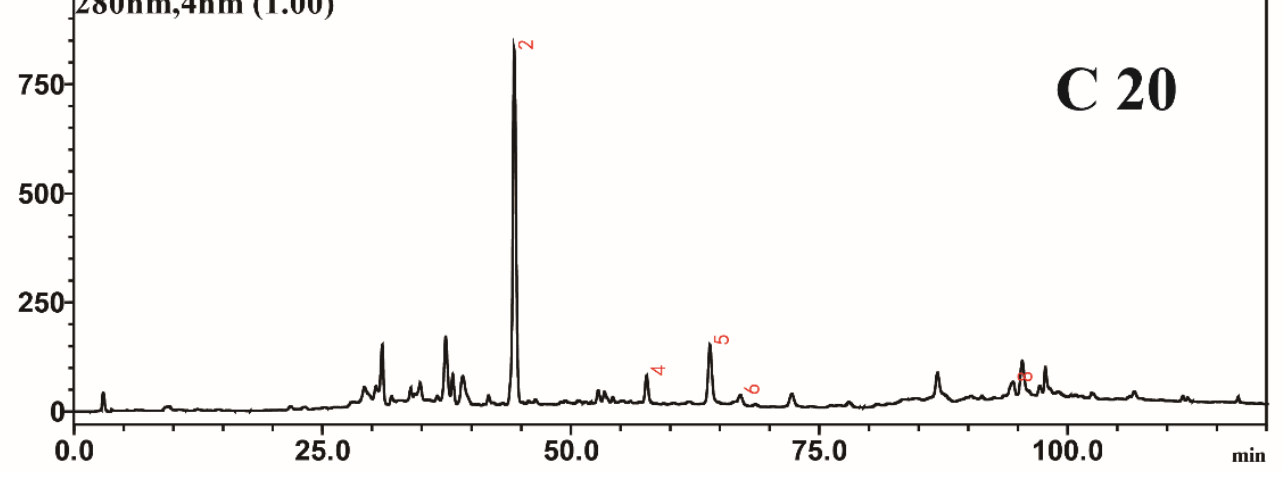



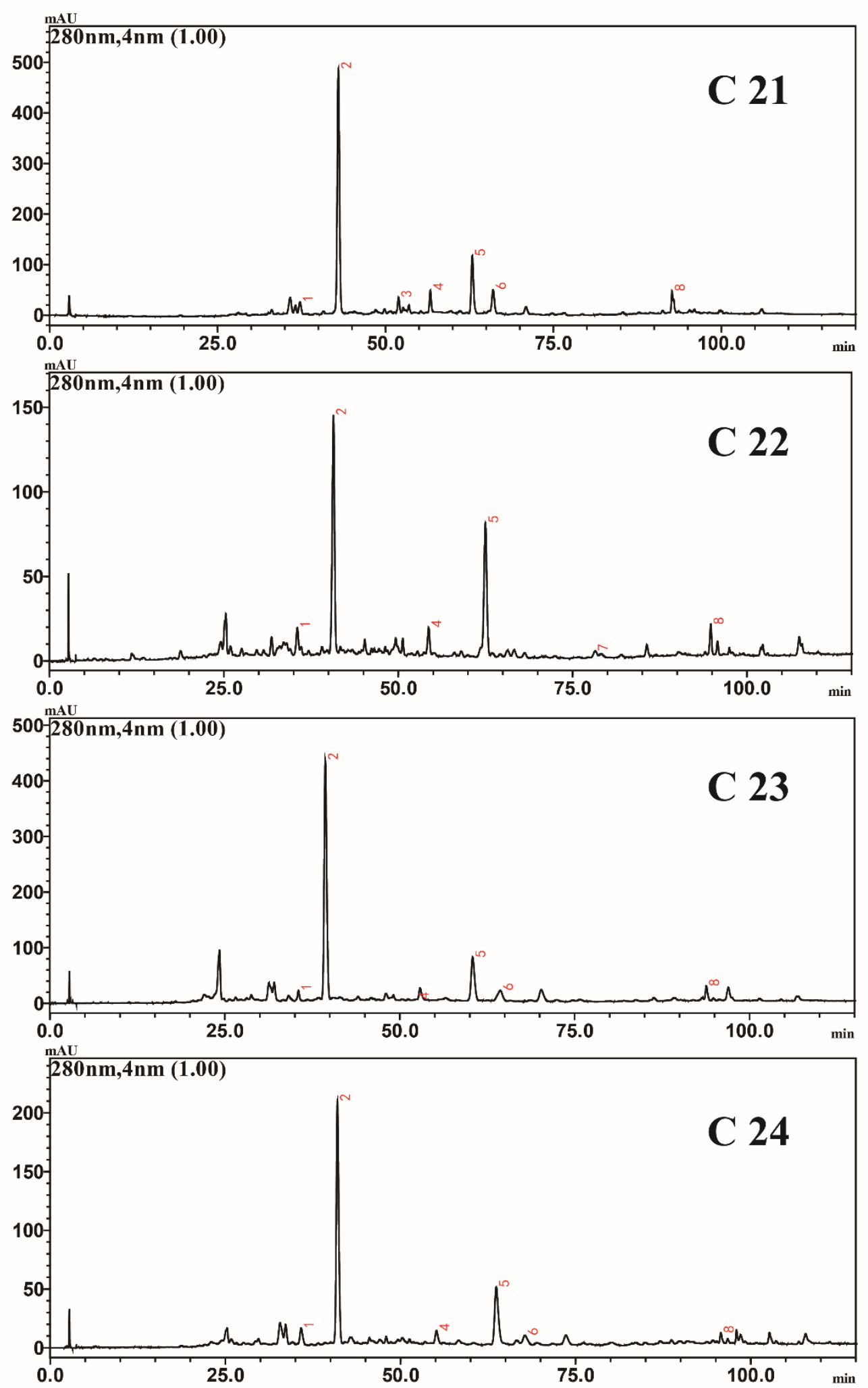

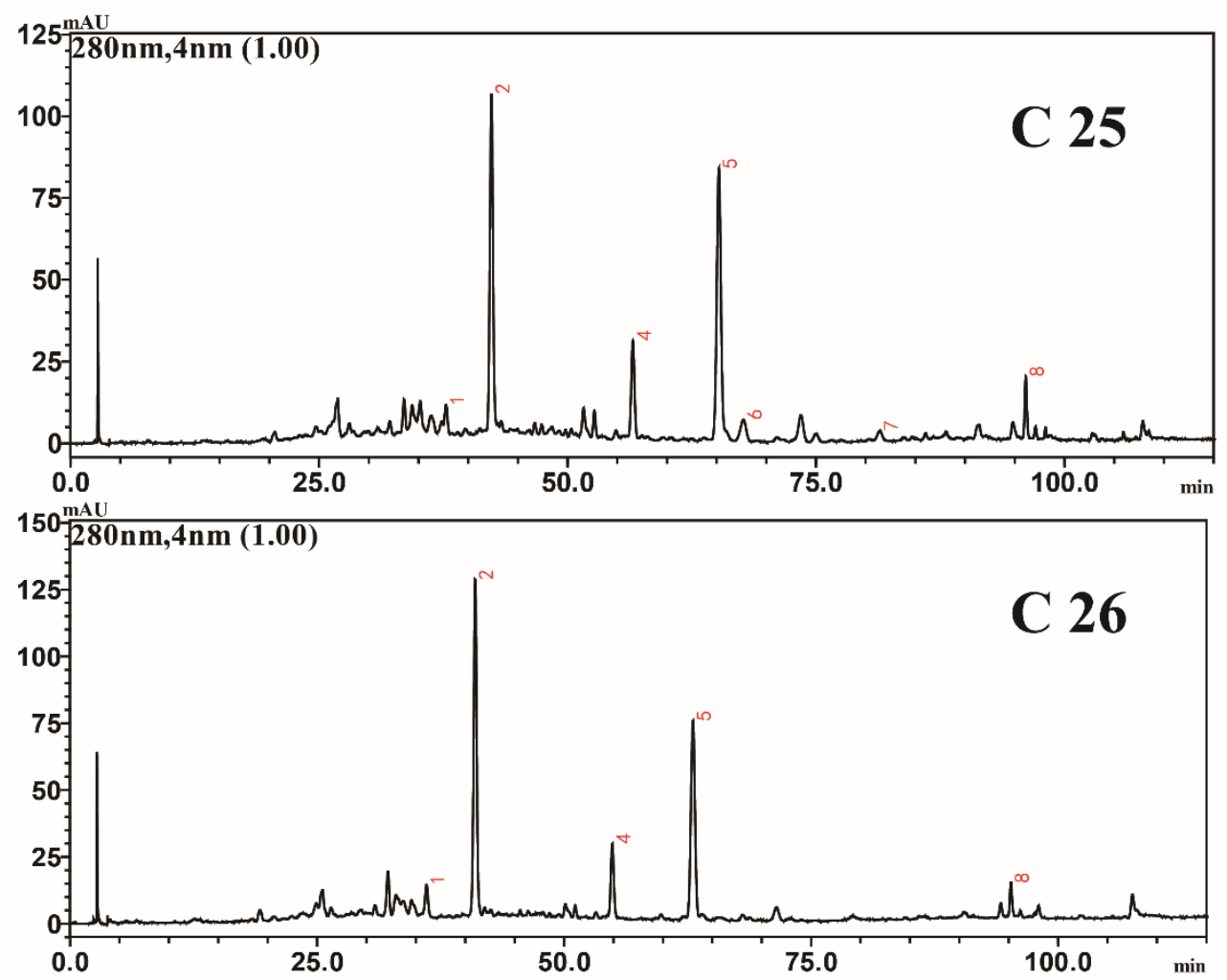

mAU

280nm,4nm (1.00)

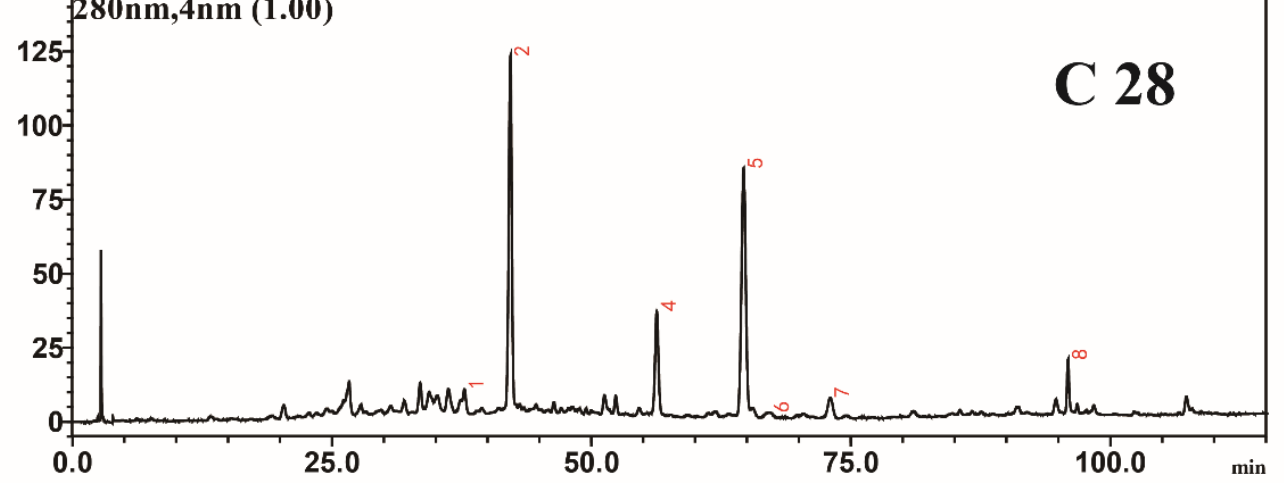



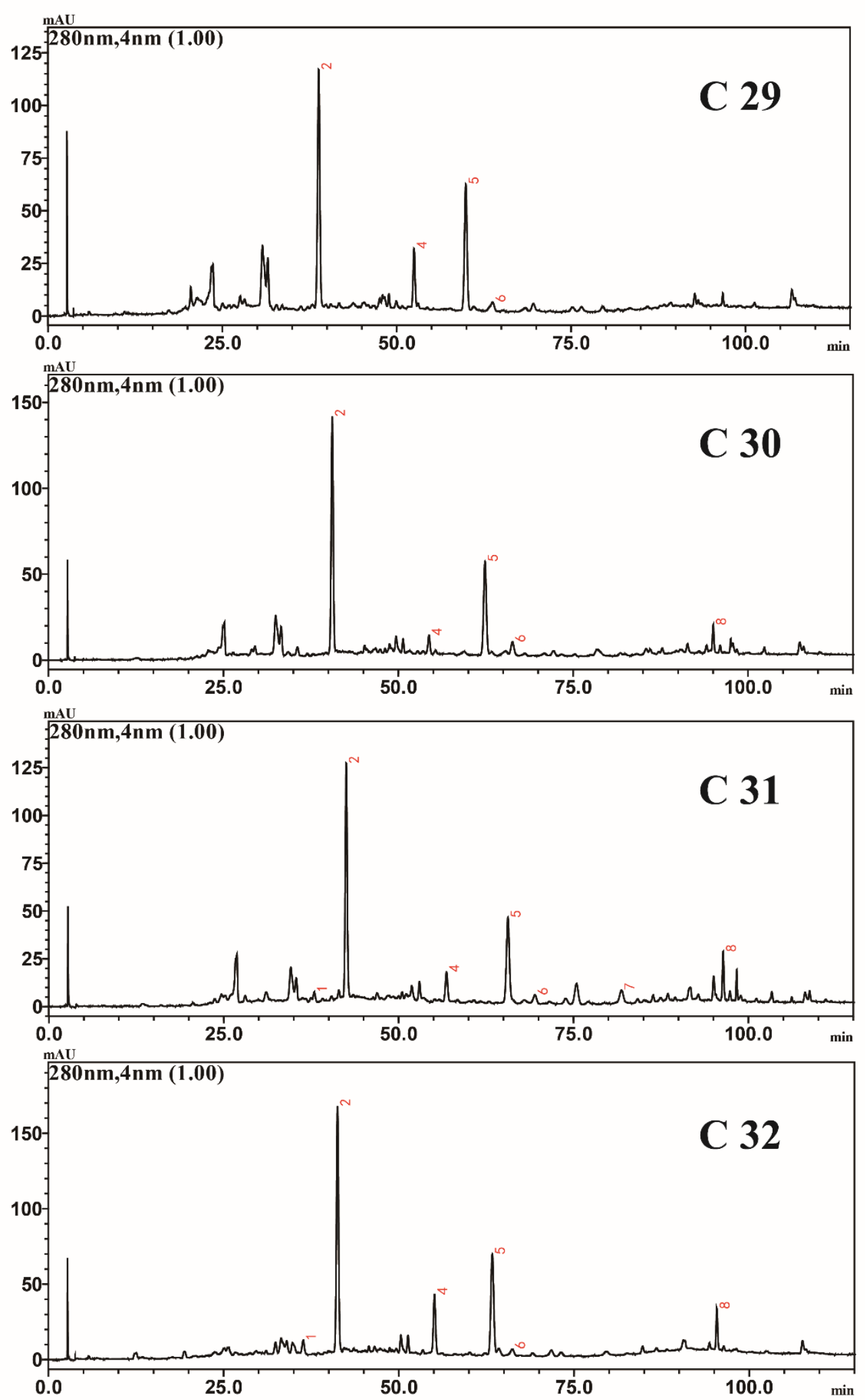

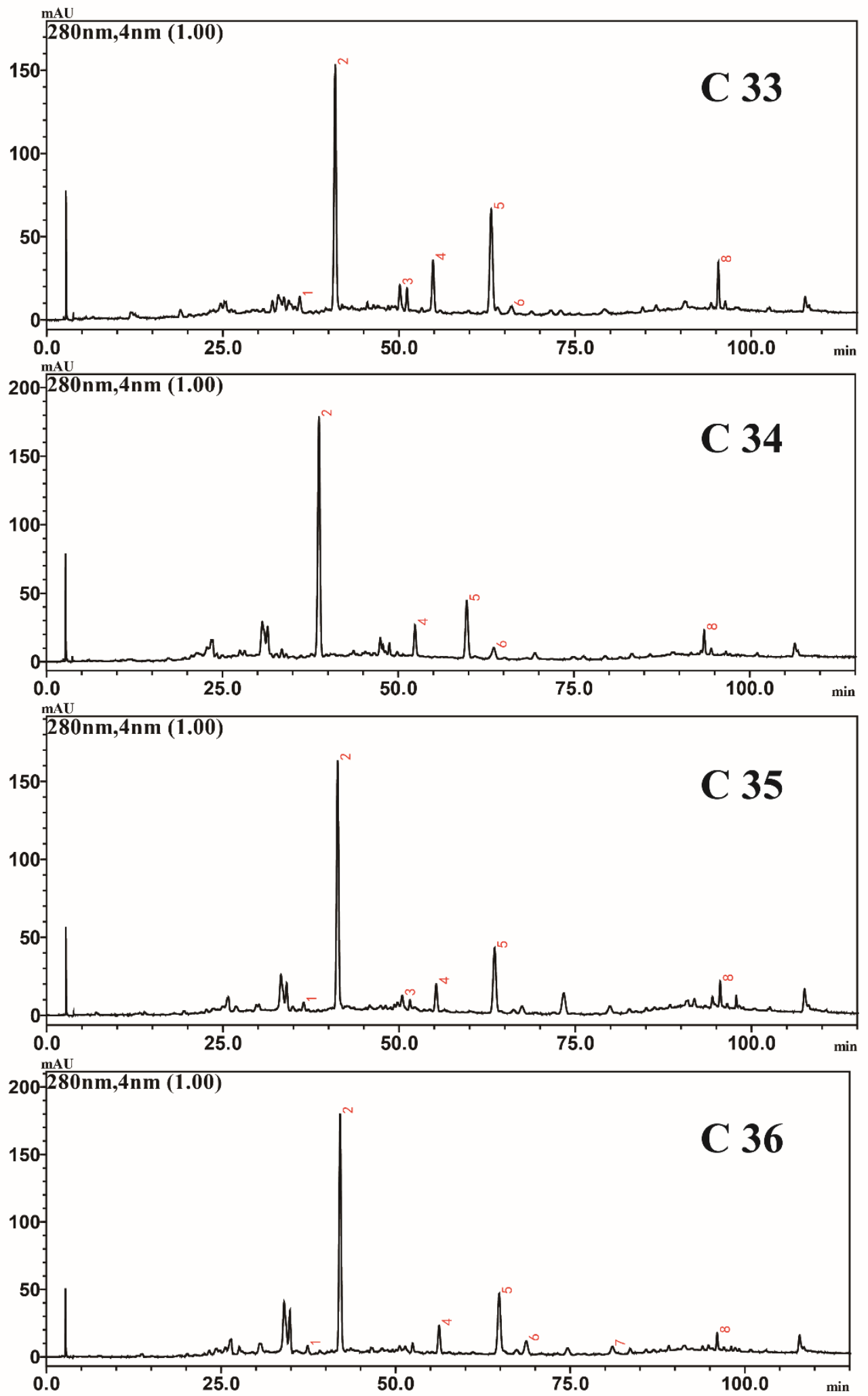

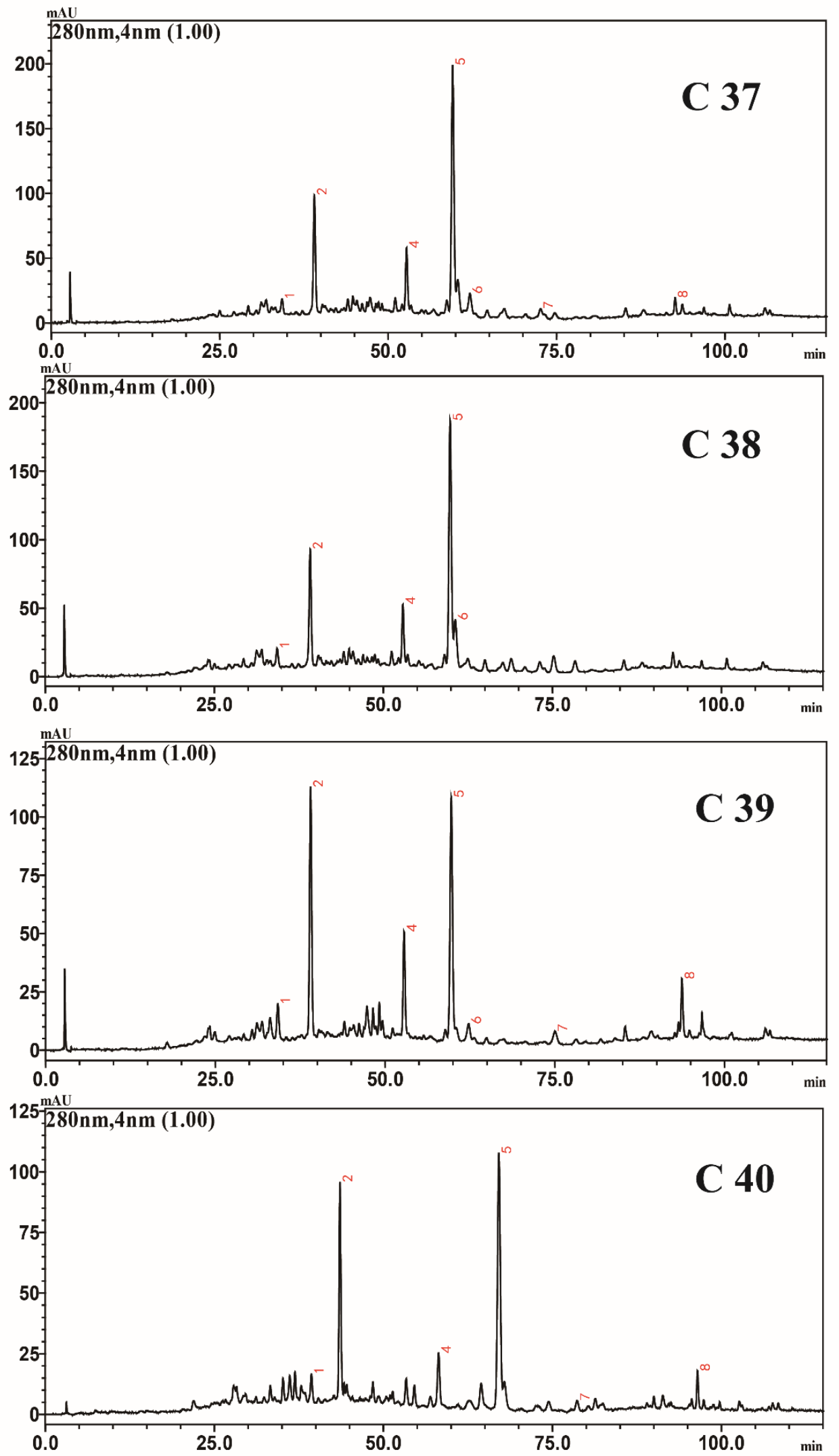

Supplementary Figure 6. HPLC profiles of raw linden honeys

Note: $\mathrm{C} 1$ to $\mathrm{C} 40$ represent sample 1 to 40 . 